\title{
Multi-machine analysis of turbulent transport in helical systems via gyrokinetic simulation
}

\section{AUTHOR(S):}

Ishizawa, A.; Kishimoto, Y.; Watanabe, T.-H.;

Sugama, H.; Tanaka, K.; Satake, S.; Kobayashi, S.; Nagasaki, K.; Nakamura, Y.

\section{CITATION:}

Ishizawa, A.... [et al]. Multi-machine analysis of turbulent transport in helical systems via gyrokinetic simulation. Nuclear Fusion 2017, 57(6): 066010.

\section{ISSUE DATE:}

2017-06

URL:

http://hdl.handle.net/2433/261144

\section{RIGHT:}

This is the Accepted Manuscript version of an article accepted for publication in Nuclear Fusion. IOP Publishing Ltd is not responsible for any errors or omissions in this version of the manuscript or any version derived from it. The Version of Record is available online at https://doi.org/10.1088/1741-4326/aa6603.; This is not the published version. Please cite only the published version.; この論文は出版社版でありません。引用の際には出版社版をご確認で利用ください。 


\title{
Multi-machine analysis of turbulent transport in helical systems via gyrokinetic simulation
}

\author{
A. Ishizawa, Y. Kishimoto T.-H. Watanabe ${ }^{1}$, H. Sugama ${ }^{2}$, K. \\ Tanaka $^{2}$, S. Satake ${ }^{2}$, S. Kobayashi ${ }^{3}$, K. Nagasaki ${ }^{3}$, and Y. \\ Nakamura \\ Graduate School of Energy Science, Kyoto University, Uji, Kyoto, 611-0011, Japan. \\ ${ }^{1}$ Department of Physics, Nagoya University, Nagoya, 464-8602, Japan \\ ${ }^{2}$ National Institute for Fusion Science, Toki, 509-5292, Japan \\ ${ }^{3}$ Institute of Advanced Energy, Kyoto University, Uji, Kyoto, 611-0011, Japan. \\ E-mail: ishizawa@energy.kyoto-u.ac.jp
}

\begin{abstract}
We have investigated drift-wave instability and nonlinear turbulent transport in two configurations with different magnetic field structure by means of electromagnetic gyrokinetic simulations. Here, one is the neo-classically optimized Large Helical Device (LHD) plasma and the other is the Heliotron J (HJ) plasma. First, we present that the validation against the turbulent transport in the LHD plasma is successful, and that the neoclassically optimized configuration has smaller turbulent transport. Second, the neoclassical optimization through an enhanced toroidal mirrorratio, which is a capability of non-axisymmetric plasma, is found to improve the turbulent transport in the HJ plasma, which is qualitatively consistent with the observation in the HJ. Hence, the neoclassical optimization reduces the turbulent transport in both the LHD and the HJ plasmas. Third, as a trial in evaluating the performance of helical system designed with different concepts for stability, we compared turbulent transport in these plasmas and found that both the mixing length estimated diffusion and nonlinear turbulent transport of the HJ plasma are smaller than those of the LHD plasma in gyro-Bohm units. The significant difference is stronger zonal flows in the HJ plasma than the LHD plasma.
\end{abstract}

PACS numbers: $52.35 . \mathrm{Ra}, 52.65 . \mathrm{Tt}, 52.25 . \mathrm{Fi}, 52.35 . \mathrm{Mw}$ 


\section{Introduction}

Stellarator and heliotron plasmas have been studied for optimization by reducing MHD instabilities and by improving neoclassical transport properties [1]. The Wendelstein 7$\mathrm{X}(\mathrm{W} 7-\mathrm{X})$ is optimized against neoclassical transport as well as MHD stability $[2,3,4]$, and the Heliotron $\mathrm{J}(\mathrm{HJ})$ is optimized against MHD stability by producing the magnetic well [5]. The Large Helical Device (LHD) has lower neoclassical transport for the inward-shifted magnetic axis configuration, while it has better MHD stability for the outward-shifted one $[6,7]$. It has been pointed out in the nonlinear MHD simulation that the $\beta$-value can be larger exceeding the critical value estimated from linear MHD stability due to the local flattening of pressure profile around rational surfaces, which is consistent with the LHD experiment aiming at high- $\beta$ [8]. In addition to the aspect of neoclassical transport and MHD stability, recently, optimization against turbulent transport becomes a hot topic $[9,10,11,12,13,14,15,16,17,18,19]$. In the analysis of the LHD plasma, the ITG turbulence is examined by gyrokinetic simulation using the adiabatic electron approximation, and in addition to be neoclassically optimized, the turbulence is suppressed by zonal flows in the inward-shifted magnetic axis configuration $[9,10,11,12]$. On the other hand, in W7-X the trapped electron region which is the straight section with weak magnetic field, and bad curvature region which is the corner section with strong magnetic field, are well separated, so that the trapped electron mode (TEM) can be avoided $[13,14,15,16,17]$. The Heliotron J has a similar magnetic structure with W7-X, i.e. the corner section with strong magnetic field and the straight one with weak magnetic field, and the ratio of the field strengths is called as the toroidal mirror ratio.

In this paper we investigate turbulent transport in helical plasmas in two devices, namely the LHD and the Heliotron J. One of the significant difference between these plasmas is the magnetic shear which represents the variation of the magnetic field line pitch from a magnetic surface to the next. The drift-wave and MHD instabilities are strongly influenced by the magnetic shear. The LHD has finite shear as well as the CHS[20] and Heliotron E, while the Heliotron J, W7-X, TJ-II[21, 22], and HSX[23] have weak shear. It is remarked that typical tokamaks have moderate magnetic shear, however the turbulent transport in weak shear regime is important even for tokamaks because the reversed shear configuration is a candidate of steady-state operation with large bootstrap current fraction [24]. Another difference between the LHD and the HJ plasmas is the magnetic well/hill characteristics which is the variation of magnetic field strength across the surface in terms of the normal curvature of the field line. The LHD has a magnetic hill as well as the CHS and Heliotron E, while the Heliotron J, W7-X, TJII, and HSX have magnetic wells. The magnetic well/hill is linked to the MHD instability such as the pressure driven interchange mode since the helical system confines a plasma by magnetic field without net toroidal plasma current, so that the current driven MHD modes are not important. Thus, the suppression of the MHD instability by producing the magnetic well is an important optimization for helical systems. 
In order to evaluate turbulent transport in helical plasmas, the gyrokinetic modeling and simulation are of importance and is a great challenge in computational science because of complicated kinetic ion and electron dynamics interacting with the electromagnetic perturbation in complex three-dimensional non-axisymmetric magnetic structures. Here, the kinetic electron dynamics is of specific importance since the effect of trapped electrons enhances the growth rate of the drift-wave instability. In order to perform such a simulation while accurately capturing the mode structure along a field line, a large number of grid-points is needed, and thus the simulations require much more computational resources than for comparable simulations of tokamak plasmas. This is the reason why most of the nonlinear gyrokinetic simulations of helical systems are limited to the adiabatic electron response approximation $[12,13,17,25,26]$, and nonlinear gyrokinetic simulations including kinetic electrons for helical systems have been just started [27, 28, 29, 30].

In our simulation study, we examine four plasmas, two for the neoclassically optimized LHD and two for the HJ configurations by using the GKV code [25, 27, 31, 32]. Two plasmas are in one discharge \#88343 in the inward-shifted configuration of LHD, and are before (LHD-L) and after (LHD-H) additional NBI heating [33]. Furthermore, two HJ plasmas are the standard configuration (HJ-ST) and the high-bumpiness configuration (HJ-HB) established by higher mirror-ratio between corner section and straight one to reduce neo-classical transport.

The paper is organized as follows. Our simulation model is described in Sec. 2. The validation of turbulent transport of energy and particles in the LHD plasmas is presented in Sec. 3. The first analysis of turbulent transport in Heliotron J (HJ) plasma is reported in Sec. 4. Comparison between them are discussed in Sec. 5. Summary is given in Sec. 6.

\section{Gyrokinetic simulation model}

Here, we briefly describe the gyrokinetic simulation model used in our work. We consider micro-turbulence in a flux tube represented by the coordinates $(x, y, z)$, where $x=\frac{q\left(\psi_{0}\right)}{B_{0} r\left(\psi_{0}\right)}\left(\psi-\psi_{0}\right), y=\frac{-r\left(\psi_{0}\right)}{q\left(\psi_{0}\right)}\left(\alpha-\alpha_{0}\right)$, and $z=\theta,(\psi, \theta, \zeta)$ is a flux coordinate system, $\alpha=\zeta-q(\psi) \theta$ is the magnetic field line label, $\psi$ is the magnetic flux, $\theta$ is the poloidal angle, and the tube is located on a field line with $\psi=\psi_{0}$ and $\alpha=\alpha_{0}$. The gyro-center distribution function for a species $s$ is divided into the Maxwellian and the perturbed parts $f_{s}\left(x, y, z, v_{\|}, \mu\right)=F_{M s}+\delta f_{s}$, where $F_{M s}=\frac{n_{0}}{\left(2 \pi T_{s} / m_{s}\right)^{3 / 2}} \exp \left(-\frac{m_{s} v_{\|}^{2}}{2 T_{s}}-\frac{\mu B}{T_{s}}\right)$. The gyrokinetic equation for perturbed ion and electron gyro-center distribution functions $\delta f_{s \mathbf{k}_{\perp}}$, the gyrokinetic Poisson equation for electrostatic potential $\phi_{\mathbf{k}_{\perp}}$, and gyrokinetic Ampère's law for parallel component of perturbed vector potential $A_{\| \mathbf{k}_{\perp}}$ are

$$
\begin{aligned}
& \frac{\partial \delta f_{s \mathbf{k}_{\perp}}}{\partial t}+v_{T s} v_{\|}\left(\mathbf{b}_{s}^{*} \cdot \nabla \delta f_{s}\right)_{\mathbf{k}_{\perp}}-v_{T s} \mu \mathbf{b} \cdot \nabla B \frac{\partial \delta f_{s \mathbf{k}_{\perp}}}{\partial v_{\|}} \\
& =-i \mathbf{v}_{d s} \cdot \mathbf{k}_{\perp}\left(\delta f_{s \mathbf{k}_{\perp}}+\frac{q_{s}}{T_{s}} F_{M s} \phi_{\mathbf{k}_{\perp}} J_{0 s}\right)-\left(\tilde{\mathbf{v}}_{E s} \cdot \nabla \delta f_{s}\right)_{\mathbf{k}_{\perp}}
\end{aligned}
$$




$$
\begin{aligned}
& \quad+i \mathbf{v}_{* s} \cdot \mathbf{k}_{\perp} \frac{q_{s}}{T_{s}} F_{M s}\left(\phi_{\mathbf{k}_{\perp}}-v_{T s} v_{\|} A_{\| \mathbf{k}_{\perp}}\right) J_{0 s}+v_{T s} v_{\|} \frac{q_{s}}{T_{s}} F_{M s} E_{\| s \mathbf{k}_{\perp}}+C_{s}, \\
& \lambda_{D i}^{2} k_{\perp}^{2} \phi_{\mathbf{k}_{\perp}}=\sum_{s} q_{s} \delta n_{s \mathbf{k}_{\perp}}^{(p)} \\
& k_{\perp}^{2} A_{\| \mathbf{k}_{\perp}}=\frac{\beta_{i}}{2} \sum_{s} q_{s} v_{T s} \delta u_{s \mathbf{k}_{\perp}}^{(p)}
\end{aligned}
$$

respectively, where $\rho_{s}=v_{T s} / \Omega_{s}$ and $\Omega_{s}=q_{s} B / m_{s} c, E_{\| s \mathbf{k}_{\perp}}=-\left(\mathbf{b}_{s}^{*} \cdot \nabla J_{0 s} \phi\right)_{\mathbf{k}_{\perp}}-$ $\frac{\partial}{\partial t} A_{\| \mathbf{k}_{\perp}} J_{0 s}, \delta n_{s \mathbf{k}_{\perp}}^{(p)}=\int \delta f_{s \mathbf{k}_{\perp}} J_{0 s} d^{3} v-\frac{q_{s}}{T_{s}}\left(1-\Gamma_{0 s}\right) \phi_{\mathbf{k}_{\perp}}, \delta u_{s \mathbf{k}_{\perp}}^{(p)}=\int v_{\|} \delta f_{s \mathbf{k}_{\perp}} J_{0 s} d^{3} v$, are the parallel component of the perturbed electric field, the perturbed density measured at the particle position, and the parallel component of perturbed velocity measured at the particle position, respectively. Nonlinear terms are included in the convective derivative by perturbed the $\mathrm{E} \times \mathrm{B}$ flow $\left(\tilde{\mathbf{v}}_{E s} \cdot \nabla f\right)_{\mathbf{k}_{\perp}}=\left[\phi J_{0 s}, f\right]_{\mathbf{k}_{\perp}}$ and the parallel component of spatial gradient including the magnetic perturbation $\left(\mathbf{b}_{s}^{*} \cdot \nabla f\right)_{\mathbf{k}_{\perp}}=$ $\mathbf{b} \cdot \nabla f_{\mathbf{k}_{\perp}}+\left(\tilde{\mathbf{b}}_{s} \cdot \nabla f\right)_{\mathbf{k}_{\perp}}=\mathbf{b} \cdot \nabla f_{\mathbf{k}_{\perp}}-\left[A_{\|} J_{0 s}, f\right]_{\mathbf{k}_{\perp}}$, where $\tilde{\mathbf{b}}_{s}=-\mathbf{b} \times i \mathbf{k}_{\perp} A_{\| \mathbf{k}_{\perp}} J_{0 s}$. The unit vector of the equilibrium magnetic field is $\mathbf{b}=\mathbf{B}_{0} / B_{0}$, and $\mathbf{b}_{s}^{*}=\mathbf{b}+\tilde{\mathbf{b}}_{s}$ includes the perturbed part of the magnetic field. The subscript 0 of the equilibrium magnetic field is omitted below. Poisson bracket and the zeroth order Bessel function are $[f, g]_{\mathbf{k}_{\perp}}=\sum_{\mathbf{k}_{\perp}^{\prime}, \mathbf{k}_{\perp}^{\prime \prime}} \delta_{\mathbf{k}_{\perp}, \mathbf{k}_{\perp}^{\prime}+\mathbf{k}_{\perp}^{\prime \prime}} \mathbf{b} \cdot \mathbf{k}_{\perp}^{\prime} \times \mathbf{k}_{\perp}^{\prime \prime} f_{\mathbf{k}_{\perp}^{\prime}} g_{\mathbf{k}_{\perp}^{\prime \prime}}$ and $J_{0 s}=J_{0}\left(\rho_{s} k_{\perp}\right)$ respectively. The magnetic drift, the diamagnetic drift, and the perturbed $\mathrm{E} \times \mathrm{B}$ drift velocities are $\mathbf{v}_{d s}=\frac{1}{q_{s} B} \mathbf{b} \times\left(\mu \nabla B+m_{s} v_{\|}^{2} \mathbf{b} \cdot \nabla \mathbf{b}\right), \mathbf{v}_{* s}=\frac{T_{s}}{q_{s} B} \mathbf{b} \times \nabla \ln F_{M s}$, and $\tilde{\mathbf{v}}_{E s \mathbf{k}_{\perp}}=$ $-\frac{1}{B}\left(i \mathbf{k}_{\perp} \phi_{\mathbf{k}_{\perp}} J_{0 s}\right) \times \mathbf{b}$, respectively. The collision operator is denoted by $C_{s}\left(h_{s \mathbf{k}_{\perp}}\right)$, where $h_{s \mathbf{k}_{\perp}}=\delta f_{s \mathbf{k}_{\perp}}+\frac{q_{s}}{T_{s}} \phi_{\mathbf{k}_{\perp}} J_{0 s} F_{M s}$ is the non-adiabatic part of the perturbed part of the gyro-center distribution function. The normalization used in the equations are $\left(t v_{T i} / R_{0}, \mathbf{k}_{\perp} \rho_{T i}, v_{\|} / v_{T s}, F_{M s} v_{T s}^{3} / n_{0}, \delta f_{s} R_{0} v_{T s}^{3} /\left(\rho_{T i} n_{0}\right), e \phi R_{0} /\left(\rho_{T i} T_{i}\right), A_{\|} R_{0} /\left(\rho_{T i}^{2} B_{0}\right)\right.$, $\left.m_{s} / m_{i}, T_{s} / T_{i}, n / n_{0}, B / B_{0}, q_{s} / e, \lambda_{D i} / \rho_{T i}\right) \rightarrow\left(t, \mathbf{k}_{\perp}, v_{\| s}, F_{M s}, \delta f_{s}, \phi, A_{\|}, M_{s}, T_{s}, n, B\right.$, $\left.q_{s}, \lambda_{D i}\right)$, where $\lambda_{D i}=\sqrt{T_{i} /\left(4 \pi e^{2} n_{0}\right)}$ and $v_{T s}=\sqrt{T_{s} / m_{s}}$, and the leading order term in the Larmor radius expansion $\rho_{i}$ is written as $\rho_{T i}=v_{T i} / \Omega_{i}$. The polarization effects due to finite Larmor radius is represented by $1-\Gamma_{0 s}$ term in the Poisson equation through $n_{s \mathbf{k}_{\perp}}^{(p)}$, where $\Gamma_{0 s}=e^{-\rho_{s}^{2} k_{\perp}^{2}} I_{0}\left(\rho_{s}^{2} k_{\perp}^{2}\right)$ and $I_{0}$ is the zeroth order modified Bessel function. Temperature and density gradients are assumed to be uniform in local simulations and in the radial direction $x$, and the gradients are represented in terms of density scale length $L_{n}=-(d \ln n / d x)^{-1}$ and temperature scale lengths $L_{T s}=-\left(d \ln T_{s} / d x\right)^{-1}$.

\section{Turbulent transport in LHD plasma}

The LHD plasma has ten periods in the toroidal direction as shown in the top-view of a magnetic surface of the LHD plasma (Fig. 1). In the analysis of LHD, we investigate plasmas before and after an additional NBI heating in the shot No. 88343. Before and after the additional NBI, the density profiles are similar, but the electron and ion temperatures are doubled as shown in Fig. 2. We call the low-temperature state as LHD-L $\left(t=1.8[s], T_{i 0}=1.7[\mathrm{keV}]\right)$ and the high-temperature state as LHD-H $(t=2.2[s]$, $\left.T_{i 0}=3.9[\mathrm{keV}]\right)$. The $q$ profile represents the finite magnetic shear in Fig. 2. The typical 
non-dimensional parameters of the LHD-H and LHD-L plasmas are shown in Table 1. The profiles of magnetic field strength $B$, the square of perpendicular wavenumber $k_{\perp}^{2}$, and the magnetic drift frequency $\omega_{d i}$ along the field line at $\rho=0.68$ of LHD-L and at $\rho=0.65$ of LHD-H plasmas are shown in Fig. 3. The square of perpendicular wavenumber $k_{\perp}^{2}$ increases along the field line in the LHD plasma because of finite magnetic shear. The magnetic drift frequency is negative around the bad curvature region $z=0$, where the outside of the torus, so that instabilities appear at the bad curvature region.

\subsection{Linear stability}

Here, we examine the drift-wave instability of the LHD plasmas by solving the linearized gyrokinetic equations numerically. We found that the LHD plasmas are unstable against the ion temperature gradient (ITG) mode. Although our simulations include magnetic perturbation, the kinetic ballooning mode (KBM) and the micro-tearing mode (MTM) do not appear, because $\beta$ is small as shown in Table 1 , and electromagnetic effects are negligible in the LHD plasmas, and thus the turbulence is predominantly electrostatic. Figure 4 shows the linear growth rate of the instability as a function of the minor radius $\rho$ and of the binormal wavenumber $k_{y}$. The ITG mode is unstable, and the trapped electron modes (TEMs) do not appear at $\rho \leq 0.83$ because of the very low density gradient $R_{0} / L_{n} \ll 1$. In the LHD-L and LHD-H plasmas, the kinetic electron (KE) effect enhances the growth rate from the one obtained by using the adiabatic electron response approximation (AE). The enhancement of the growth rate is crucial to the validation as shown in the next subsection.

The mode structure along the magnetic field line is represented by the electrostatic potential profile of the ITG mode in Fig. 5, and the profile of the mode in a typical tokamak plasma using the Cyclone base case (CBC) parameter is also shown as a reference $[27,30]$. The structure of ITG mode in LHD and in CBC plasmas has one peak at the bad curvature region $z=0$. The profile of the ITG mode in LHD expands as wide as that in CBC because of finite magnetic shear of these plasmas. In addition, the mode structure has oscillations because of the helical ripples.

\subsection{Nonlinear analysis}

We performed nonlinear gyrokinetic simulations of turbulence driven by the instabilities described in the previous subsection in the LHD-L and LHD-H plasmas. The typical profile of the ITG turbulence on the cross section at $z=0$ is shown in Fig. 6. The turbulent fluctuation of the ITG mode is elongated in the radial direction and is also sheared by zonal flows.

Here, we present our results on the validation study by evaluating the turbulent energy transport of ions and electrons as well as the turbulent particle transport. The turbulent energy fluxes are shown in Fig. 7 (a) as a function of the minor radius. The symbols are from the gyrokinetic simulations, $Q_{s}^{\text {Turb }}$, and the curves are from the 
experiments subtracted by the neoclassical transport, $Q_{s}^{\mathrm{Exp}}-Q_{s}^{\mathrm{Neo}}$, calculated by the FORTEC-3D code [34], where $s$ denotes the species. In the LHD-L plasma $(t=1.8[s]$, $\left.T_{i 0}=1.6[\mathrm{keV}]\right)$, the electron energy flux from the simulation is in good agreement with the experimental observation at $\rho>0.65$. The ion energy flux from the simulation is also in good agreement with the experiment at $\rho>0.65$. On the other hand, the flux from the simulation is much smaller than that from the experiment because of weak ITG instability at $\rho<0.65$. When we artificially increase the temperature gradient about $20 \%$, the linear growth rate of the ITG mode at $\rho=0.57$ is close to the one at $\rho=0.68$, where the simulation reproduces the experimental result, and thus it is expected that the energy flux at $\rho=0.57$ can be reproduced by increasing the temperature gradient about $20 \%$. The influence of the error bars of the observation is discussed later. In the LHD-H plasma $\left(t=2.2[s], T_{i 0}=3.9[\mathrm{keV}]\right)$, the electron energy flux from the simulation is in good agreement with the experimental observation. On the other hand, the ion energy flux from the simulation overestimates the experimental observation. When we reduce the temperature gradient about 20\%, then we have an agreement between the simulation and the experimental results [29]. Here, the turbulent energy flux is compared with neoclassical energy flux in Fig. 7 (b). The turbulent energy flux is much larger than the neoclassical energy flux at $\rho=0.83$ both in the LHD-H and LHD-L plasmas. The turbulent electron energy flux is comparable to the neoclassical electron energy flux, while the turbulent ion energy flux is larger than the neoclassical ion energy flux at $\rho=0.65$ in the LHD-H plasma and at $\rho=0.68$ in the LHD-L plasma. The neoclassical energy flux is larger than the turbulent energy flux at $\rho=0.61$ in the LHD-L plasma, and they are comparable at $\rho=0.46$ in the LHD-H plasma. Thus, the turbulent transport dominates around the edge region, while the neoclassical transport is important in the core region.

The turbulent particle flux and the neoclassical particle flux are shown in Fig. 8. The curves represent the neoclassical particle flux from the FORTEC-3D code, and symbols represent the turbulent particle flux from the gyrokinetic simulation. The turbulent particle flux is negative and it has pinch effects at $\rho>0.5$ in the LHD-H plasma. This is explained by the balance between the turbulent particle flux and the neoclassical particle flux $\Gamma^{\text {Turb }}+\Gamma^{\text {Neo }} \approx 0$, which is expected because the discharge of the LHD plasma (discharge No. 88343) is in a steady state and there is little fueling at $t=1.8$ and $2.2[s]$. On the other hand, the turbulent particle flux is positive at $\rho<0.5$ of the LHD-H and in the LHD-L plasmas, and the balance between the turbulent transport and the neoclassical one is clearly violated. This can not be explained even by taking the error bars described below into account, and we need to study the particle flux in future work.

Here, we summarize the validation of turbulent transport against the experimental LHD plasma. The ion and electron temperature gradients obtained from the flux-matching method for the LHD-H and LHD-L plasmas are in agreement with experimental observations[33] within a 20\% error. The error would be reduced by introducing the influence of the global radial electric field shear to the GKV code, 
because the LHD-H plasma is in the electron root of the ambipolarity condition with strong global radial electric field at $0.4<\rho<0.9$ in contrast to the LHD-L plasma which is in the ion root with weak radial electric field. It is noted that the observation error of the ion temperature is presented in Fig. 2, and it is about $4 \%$ at $0.45<\rho<0.85$ in LHD-H and about $2 \%$ at $0.5<\rho<0.85$ in LHD-L. The error of the observation can cause about $10 \%$ error in the gradient of the temperature. This error can result in the error of turbulent energy flux about 30\%, and the error of the neoclassical transport is about three times of the error of temperature. It is also noted that, in the validation against LHD plasmas, there is no short-fall problem at the edge region, which has been observed in some tokamaks $[35,36,37]$. A possible explanation for this is that the typical discharge of LHD plasma is not unstable against the TEM but unstable against the ITG mode from the core to the edge due to the flat density profile.

Here, we compare the turbulent transport in the LHD-L and LHD-H plasmas. Table 1 shows that the energy diffusion coefficients $\chi_{i}$ and $\chi_{e}$ in the LHD-L plasma are smaller than those in the LHD-H plasma, in spite of the fact that the mixing length estimated diffusion $\gamma / k_{\perp}^{2}$ in the LHD-L plasma is larger than that in the LHD-H plasma in Fig. 9 , which shows $\gamma / k_{\perp}^{2}$ as a function of the binormal wavenumber $k_{y} \rho_{T i}$ at $\rho=0.65$ of the LHD-H and at $\rho=0.68$ of the LHD-L plasmas. It is noticed that the most unstable mode has $k_{x}=0$ in the linear calculation, so that $k_{\perp}^{2}=k_{y}^{2}$. We expect that the difference between diffusion coefficients is due to the zonal flows. Figure 10 shows the spectrum of the square of the electrostatic potential $\left\langle\left|\phi_{k}\right|^{2}\right\rangle$ averaged over a time period in the statistical steady state as a function of the binormal wavenumber $k_{y}$, the radial wavenumber $k_{x}$, and in $\left(k_{x}, k_{y}\right)$ space. The amplitude of the ITG mode and that of zonal flow are comparable in $\left(k_{x}, k_{y}\right)$ space, and is rather broad in the radial wavenumber direction $k_{x}$ in the LHD-L plasmas. Comparison of the spectra between the LHD-L and LHD-H plasmas in $k_{y}$ implies that the partition of zonal flow is larger in the LHD-L plasma than that in the LHD-H plasma. The ratio of the zonal-flow component to the ITG mode is $\left\langle\left|\phi_{\mathrm{ZF}}\right|^{2}\right\rangle /\left\langle\left|\phi_{\mathrm{ITG}}\right|^{2}\right\rangle=\left\langle\left|\phi_{k y=0}\right|^{2}\right\rangle /\left\langle\left|\phi_{k y=0.23}\right|^{2}\right\rangle=1.2$ in the LHD-L and $\left\langle\left|\phi_{k y=0}\right|^{2}\right\rangle /\left\langle\left|\phi_{k y=0.17}\right|^{2}\right\rangle=0.74$ in the LHD-H, and thus the ratio is larger in the LHD-L than the LHD-H. This explains the lower turbulent transport in the LHD-L plasma than in the LHD-H plasma. A possible explanation is the zonal flow response in Fig. 11 which shows the zonal flow damping history in the LHD-L and LHD-H plasmas for several radial wavenumbers $k_{x} \rho_{T i}=0.1,0.2,0.3$, and 0.4 . The longer damping time scale of zonal flow in the LHD-L plasma than in the LHD-H plasma in Fig. 11 contributes to larger zonal flows in the LHD-L plasma. By assuming that the nonlinear drive from the turbulence in the LHD-L and LHD-H plasmas are similar, it explains the larger zonal flows in the LHD-L, and thus the smaller $\chi_{i}$ and $\chi_{e}$ of the LHD-L plasma compared to the LHD-H plasma. This is similar to the mechanism of turbulent transport reduction in the inward-shifted LHD plasma shown in Refs. $[9,10,11,12]$, because the magnetic axis of the LHD-L plasma is more inward-shifted than the LHD-H plasma, and the magnetic field strength profile of the LHD-L plasma implies lower neoclassical transport than in the LHD-H plasma in Fig. 3. 


\section{Turbulent transport in Heliotron J plasma}

The Heliotron $\mathrm{J}(\mathrm{HJ})$ plasma has four periods in the toroidal direction and it consists of four straight sections and four corner sections as shown in the top-view of a magnetic surface in Fig. 12. In this analysis, the standard (HJ-ST) and a strong toroidal ripple (high-bumpiness) configurations (HJ-HB) are examined. Figure 13 shows the profiles of the safety factor $q$, density, and temperatures of the HJ plasmas. The $q$ profile is very flat with $q \approx 1.8$, so that the magnetic shear is very weak. The typical non-dimensional parameters of the HJ-ST and HJ-HB plasmas are summarized in Table 2. Figure 14 shows the profiles of magnetic field strength $B$, the square of perpendicular wavenumber $k_{\perp}^{2}$, and the magnetic drift frequency $\omega_{d i}$ along the field line at $\rho=0.5$ of HJ-ST and at $\rho=0.5$ of HJ-HB plasmas. The magnetic field strength ratio between the corner and straight sections are indeed enhanced in the HJ-HB compared with HJ-ST. The strength of mirror-ratio is measured by the variation of the field strength along the field line, and the high mirror-ratio configuration is considered to improve the neo-classical transport [5]. The square of perpendicular wavenumber $k_{\perp}^{2}$ increases very slowly in the HJ plasmas because of the weak magnetic shear.

\subsection{Linear stability}

Here, we examine the linear stability of drift-waves in the HJ-ST and HJ-HB plasmas. These plasmas are unstable against the ITG mode, which rotates in the ion diamagnetic direction as indicated by the negative real frequency in Fig. 15. We confirmed that the trapped electron modes do not appear at $\rho<0.6$ in the HJ plasmas.

The suppression effect of higher toroidal mirror-ratio (bumpiness) on the ITG mode is revealed by the smaller growth rate $\gamma=0.26\left[v_{T i} / R_{0}\right]$ in the HJ-HB plasma compared to $\gamma=0.4$ in the HJ-ST plasma in Fig. 15, which shows the linear growth rate of the instabilities as a function of the binormal wavenumber $k_{y} \rho_{T i}$ at $\rho=0.5$. The suppression of the ITG mode can be explained by the finite Larmor radius stabilizing effects $k_{\perp}^{2} \rho_{T i}^{2}$ through $\Gamma_{0 i}\left(k_{\perp}^{2} \rho_{T i}^{2}\right)$ and $J_{0 i}\left(k_{\perp}^{2} \rho_{T i}^{2}\right)$ in the gyrokinetic equation, Eqs. (1)-(3), because the amplitude of $k_{\perp}^{2} \rho_{T i}^{2}$ is enhanced around the bad curvature region $z=0$ in the HJ-HB plasma as shown in Fig. 14. The enhancement of $k_{\perp}^{2}$ is caused by the high magnetic field strength at the bad curvature region compared to weak magnetic field at the straight section through the metric tensor. The details of the suppression mechanism will be reported elsewhere.

We found that the mode structure in the HJ plasma is much more elongated along the field line than the one in the CBC plasma. Figure 16 shows that the electrostatic potential profile of the ITG mode along the magnetic field line is elongated. This is because the HJ plasma has weak magnetic shear. The weak magnetic shear causes very slow increase of $k_{\perp}^{2} \rho_{T i}^{2}$ along the field line as shown in Fig. 14, and it leads to slow increase of the finite Larmor radius stabilizing effects $\Gamma_{0 i}\left(k_{\perp}^{2} \rho_{T i}^{2}\right)$ and $J_{0 i}\left(k_{\perp}^{2} \rho_{T i}^{2}\right)$ in Eqs. (1)-(3) along the field line, and results in the elongated mode structure. The mode structure of the instability in the HJ plasma has a peak around the bad curvature 
region $z=0$ that is the outside of the corner part, and is elongated along the magnetic field line with oscillations. Thus, the weak magnetic shear elongates the mode structure. The stabilizing effect of the magnetic shear is confirmed by the reduction of the growth rate from $\gamma=0.4$ to $0.3\left[v_{T i} / R_{0}\right]$ by artificially increasing $\hat{s}$ from 0.023 to 0.064 in the HJ-ST plasma.

\subsection{Nonlinear analysis}

Here, we firstly report the gyrokinetic simulation of turbulent transport in the Heliotron $\mathrm{J}$ (HJ) plasma. We have performed nonlinear gyrokinetic simulations of turbulence in the HJ-ST and HJ-HB plasmas. Figure 17 shows the color map of electrostatic potential of the ITG turbulence in the HJ-ST plasma. The striped pattern exhibits the very elongated mode structure of the ITG turbulence along the field line. The electrostatic potential profile on a cross section shows that the turbulent structure is sheared by zonal flows. The strong zonal flow is clearly presented by the color map of electrostatic potential perturbation on the cross section $z=0$ in Fig. 18. The zonal flows share the ITG mode structure in the figure. The strong zonal flows are also shown by the spectra of electrostatic potential in the HJ-ST and HJ-HB plasmas as a function of $k_{y}$ in Fig. 19. The square of zonal flow potential amplitude $\left\langle\left|\phi_{k}\left(k_{y}=0\right)\right|^{2}\right\rangle$ is given by filled circles in the figure. It implies that the zonal flow energy partition is high, and thus the strong zonal flow is expected to suppress the turbulent transport. Figure 19 also shows the spectrum as a function of the radial wavenumber $k_{x}$ and in $\left(k_{x}, k_{y}\right)$ space. The ITG modes appear at $k_{y} \rho_{T i} \approx 0.35$, and strong zonal flows appear at $k_{y} \rho_{T i}=0$ in the HJ plasmas. The spectra imply that the peak of the zonal flow potential is located at $k_{x} \rho_{T i}=0.06$.

Here, we evaluate the turbulent energy transport of ions and electrons. The turbulent energy transport coefficients of the HJ-HB plasma are smaller than those of the HJ-ST plasma in Table 2. The tendency, i.e. the reduction of transport, coincides with that of linear analysis, which is the reduction of the ITG mode and of the mixing length estimated diffusion $\gamma / k_{\perp}^{2}$ shown in Fig. 15. The reduction of the transport through the suppression of the ITG mode is qualitatively consistent with the observation in the HJ experiment[38]. Since it is considered that the high toroidal mirror-ratio (bumpiness) improves the neo-classical transport in the HJ plasma, the neoclassical and turbulent transport optimizations are compatible in the HJ plasma.

\section{Discussion on comparison between turbulent transport in LHD and the one in $\mathrm{HJ}$}

The study of micro-instability and associated turbulent transport in different magnetic configurations which exhibit different response to neo-classical dynamics and MHD instability is important and even urgent to know the integrated performance of each helical system. From this viewpoint, as a trial, here we focus on two parameters, i.e. 
the magnetic shear $\hat{s}=\frac{1}{q} \frac{d q}{d r}$ and the magnetic well parameter indicating the influence of normal curvature on the Mercier index, $D_{\text {well }}=-\beta^{\prime}\left\langle\kappa_{n}\right\rangle\left\langle B_{0}^{2} /|\nabla \psi|^{2}\right\rangle$ [39], which are both related to magnetic structure and influence instabilities in different way, where $\beta^{\prime}$ is the radial gradient of the plasma beta. The parameter $D_{\text {well }}$ is linked to the averaged magnetic drift frequency $\left\langle\omega_{d}\right\rangle$ through the normal curvature of the magnetic field line $\kappa_{n}$, where $\omega_{d} \equiv \mathbf{v}_{d} \cdot \mathbf{k}_{\perp}=\omega_{d i}$. This is because $D_{\text {well }}$ is proportional to $\kappa_{n}$ which is a part of the magnetic field line curvature $\kappa=\mathbf{b} \cdot \nabla \mathbf{b}$ in the magnetic drift frequency $\omega_{d}$. The toroidal ITG mode is normally unstable when $\omega_{d} \omega_{*}>0$, where $\omega_{*}=\mathbf{v}_{*} \cdot \mathbf{k}_{\perp}$ is the diamagnetic drift frequency and is proportional to $-\beta^{\prime}$. When a plasma has a magnetic hill $D_{\text {well }}<0$, then the normal curvature is negative $\left\langle\kappa_{n}\right\rangle<0$, and thus the contribution of the normal curvature to the magnetic drift frequency $\omega_{d}$ is negative and destabilizing. This is confirmed by averaging the magnetic drift frequency in Figs. 2 and 13. We have $\left\langle\omega_{d i}\right\rangle=-7.5$ in the LHD-H, -7.4 in the LHD-L, 3.30 in the HJ-HB, and 3.31 in the HJ-ST. They are consistent with $D_{\text {well }}=-0.02$ in the LHD-H, -0.01 in the LHD-L, 0.64 in the HJ-HB, and 0.74 in the HJ-ST in Tables 1 and 2.

It can be seen that helical devices are roughly categorized into two families, one is the moderate shear and magnetic hill realized in neoclassically optimized LHD plasma, CHS, and Heliotron E, and the other the weak shear and magnetic well realized in W7-X, Heliotron J, TJ-II, and HSX. The characteristics of the former result from emphasizing the magnetic shear stabilization with axisymmetric magnetic axis, while those latter result from the non-axisymmetric magnetic-axis producing the magnetic well for optimized stability. Therefore, it is worthwhile to study the micro-instability and associated turbulent transport paying attention to the parameter region for these two families. The neo-classically optimized LHD plasma can be a representative of the former case (moderate shear, hill) and the Heliotron J plasma of the latter (weak shear, well). We compared, for the first time, the characteristics in two configurations to find a guideline in evaluating the performance of a helical system. The typical non-dimensional parameters of the LHD-H, LHD-L, HJ-ST, and HJ-HB plasmas are shown in Tables 1 and 2. It is found that many parameters of the LHD and HJ plasmas, the aspect ratio $R_{0} / a$, safety factor $q$, normalized Larmor radius $\rho_{*}$, and temperature ratio $T_{e} / T_{i}$, are similar. On the other hand, the Mercier magnetic well measure $D_{\text {well }}$ and the magnetic shear $\hat{s}$, which are tightly related to magnetic structure, are significantly different, where a positive (negative) $D_{\text {well }}$ indicates the magnetic well (hill). The LHD plasmas exhibit a magnetically "hill" with moderate magnetic shear $\hat{s}$ while the HJ plasmas a "well" with extremely small $\hat{s}$. It is noted that the normalized collision frequency $\nu^{*}$ and the density gradient length $L_{n}$ are also significantly different.

The linear growth rate of the ITG mode in the HJ-ST plasma is larger than those in the LHD (LHD-H and LHD-L) plasma as shown in Figs. 9 and 15, while the mixing length estimated diffusion $\gamma / k_{\perp}^{2}\left[v_{T i} \rho_{T i}^{2} / R_{0}\right]$ of the ITG mode in the HJ plasma is smaller than that in the LHD plasma, because the wavenumber of the most unstable ITG mode in the HJ plasma is higher than that in the LHD plasma. The turbulent energy transport coefficients $\chi_{i}$ and $\chi_{e}$ of the HJ plasma are smaller than those of the LHD plasma in terms 
of the gyro-Bohm unit $\left[v_{T i} \rho_{T i}^{2} / R_{0}\right]$ (Tables 1 and 2 ). The difference can be explained by the smaller mixing length estimated diffusion (Figs. 9 and 15) and the strong zonal flow in the HJ plasma. The amplitude of the zonal flow is controlled by two mechanisms, because the gyrokinetic equation for the zonal-flow component consists only of two parts as shown by Eq. (1) in Ref. [9]. One is the linear damping caluculated by the zonal flow damping test shown below. The other is the source term representing the nonlinear drive from instabilities. The residual level of zonal flow damping depends on $q$ and $R_{0} / a$ [9], which are similar in the LHD and HJ plasmas, and large $\nu^{*}$ of the HJ plasma may reduce the zonal flow amplitude, and thus it is expected that the residual level of the zonal flow in the HJ plasma is not larger than that in the LHD plasma. This is confirmed by the zonal flow damping history in the HJ and LHD plasmas in Figs. 11 and 20. The residual level of the zonal flows in the HJ and the LHD plasmas are similar and very small, and the damping time scales of the HJ plasmas are similar to the LHD-H plasma and smaller than that of the LHD-L plasma. These zonal flow damping results imply that the nonlinear drive is the only cause of the stronger zonal flow in the HJ plasma. It is expected that the strong nonlinear zonal flow production is caused by the mode structure of the instability like that in Ref. [40], and the elongated mode structure along the magnetic field line shown in Fig. 16 may be responsible for the strong zonal flow production. In addition, the slow decrease of the linear growth rate with $k_{x}$ in Fig. 15 can be a possible mechanism of the strong zonal flow production in the HJ plasmas, because the nonlinear zonal flow production is caused by the interaction between the most unstable mode $\left(k_{y}^{I T G}, 0\right)$ and the side-band mode $\left(-k_{y}^{I T G}, k_{x}^{\text {sideband }}\right)$ which has a finite radial wavenumber. The stronger zonal flow in the HJ plasma is a possible explanation of a higher deviation of temperature gradient from the linear threshold of the ITG mode $R_{0} / L_{T}-R_{0} / L_{T \text { crit }}$ in the HJ plasma than that in the LHD plasma shown in Tables 1 and 2 .

\section{Summary}

We investigated linear drift-wave instability and nonlinear turbulent transport in two helical systems, namely the neoclassically optimized LHD and the Heliotron J (HJ), by means of the electromagnetic gyrokinetic simulations. Linear analysis revealed that both plasmas have unstable ITG modes however TEMs are stable from the core to the edge. In the validation, the enhancement of the linear growth rate by the kinetic electron effects is crucial, and thus the turbulent transport is calculated by the nonlinear simulations including kinetic electrons. The validation of the predicted turbulent transport in LHD plasmas is successful except for an underestimate at the core region of the LHD-L. It is found that the inward-shifted configuration, which is more optimized for neoclassical transport, has smaller turbulent transport in the LHD plasma because of stronger zonal flows. It is also found that the neoclassical optimization for the HJ plasma by producing a high toroidal mirror-ratio reduces the turbulent transport because of lower ITG mode growth rate. Hence, the neoclassical optimization reduces the turbulent transport in 
both the LHD and the HJ plasmas, while the reduction mechanisms are different. When we compare the LHD plasma with the HJ plasma, the turbulent transport in the HJ plasma is smaller than that in the LHD plasma in gyro-Bohm units $\left[v_{T i} \rho_{T i}^{2} / R_{0}\right]$. This can be explained by stronger zonal flows and lower mixing length estimated diffusion in the HJ plasma.

\section{Acknowledgments}

The authors thank Drs M. Nunami, M. Nakata, and S. Maeyama for helpful discussions, and Dr J. Anderson for careful reading of the manuscript. The work is supported by MEXT Grant Nos 23561003 and 16K06941 and by the NIFS Collaborative Research Program (NIFS16KNST106 and NIFS16KNTT035). This research used computational resources of the K computer at RIKEN, the Plasma Simulator at NIFS, and the Helios at CSC-IFERC, and was supported by MEXT as Priority Issue on Post-K computer.

\section{References}

[1] Spong, D. A., Phys. Plasmas 22, 055602 (2015).

[2] Nuhrenberg, J., and Zille, R., Phys. Lett. A 114, 129 (1986).

[3] Nuhrenberg, J., Plasma Phys. Control. Fusion 52, 124003 (2010).

[4] Geiger, J., Beidler, C. D., Feng, Y., Maaberg, H., Marushchenko, N. B. and Turkin, Y., Plasma Phys. Control. Fusion 57, 014004 (2015).

[5] Wakatani, M., Nakamura, Y., Kondo, K., et.al., Nucl. Fusion 40, 569 (2000).

[6] Yamada, H., et.al., Plasma Phys. Control. Fusion 43 A55 (2001).

[7] Kaneko, O., Yamada, H., Inagaki, S., and LHD Experiment Group, Nuclear Fusion 53 (2013) 104015.

[8] Ichiguchi, K., and Carreras, B. A., Nuclear Fusion 51, 053021 (2011).

[9] Sugama, H. and Watanabe, T.-H., Phys. Rev. Lett. 94, 115001 (2005).

[10] Sugama, H. and Watanabe, T.-H., Phys. Plasmas 13, 012501 (2006).

[11] Watanabe, T.-H., Sugama, H., and Ferrando-Margalet, S., Nucl. Fusion 47, (2007) 1383.

[12] Watanabe, T.-H., Sugama, H., Margalet, S.F., Phys. Rev. Lett. 100, (2008) 195002.

[13] Xanthopoulos, P., Merz, F., Görler, T., and Jenko, F., Phys. Rev. Lett. 99, (2007) 035002.

[14] Xanthopoulos, P., Mischchenko, A., Helander, P., Sugama, H., and Watanabe, T.-H., Phys. Rev. Lett. 107, (2011) 245002.

[15] Xanthopoulos, P., Mynick, H., Helander, P., Turkin, Y., et al., Phys. Rev. Lett. 113, 155001 (2014)

[16] Helander, P., Beidler, C.D., Bird, T.M., M Drevlak, Y Feng, R Hatzky, F Jenko, R Kleiber, J H E Proll, Yu Turkin and P Xanthopoulos, Plasma Phys. Control. Fusion 54 (2012) 124009.

[17] Helander, P., Bird, T., Jenko, F., et.al., Nucl. Fusion 55, 053030 (2015).

[18] Mynick, H. E., Pomphrey, N., and Xanthopoulos, P., Phys. Rev. Lett. 105, 095004 (2010).

[19] Mynick, H., Xanthopoulos, P., Faber, B., Lucia, M., Rorvig M. and Talmadge, J. N., Plasma Phys. Control. Fusion 56094001 (2014).

[20] Shats, M. G., Toi, K., Ohkuni, K., et.al., Phys. Rev. Lett. 84, 6042 (2000).

[21] Hidalgo, C., Alejaldre, C., Alonso, A., et.al., Nucl. Fusion 45, S266 (2005).

[22] Ascasibar, E., Estrada, T., Castejon, F., et.al., Nucl. Fusion 45, 76 (2005).

[23] Faber, B. J., Pueschel, M. J., Proll, J. H. E., et.al., Phys. Plasmas 22, 072305 (2015).

[24] Kishimoto, Y., Kim, J.-Y., et.al., Plasma Phys. Control. Fusion 41, A663 (1999). 
[25] Nunami, M., Watanabe, T.-H., Sugama, H., and Tanaka, K., Plasma Fusion Res. 6, (2011) 1403001.

[26] Nunami, M., Watanabe, T.-H., Sugama, H., and Tanaka, K., Phys. Plasmas 19, (2012) 042504.

[27] Ishizawa, A., Maeyama, S., Watanabe, T.-H., Sugama, H., and Nakajima, N., Nuclear Fusion 53, (2013) 053007.

[28] Ishizawa, A., Watanabe, T.-H., Sugama, H., Maeyama, S., and Nakajima, N., Phys. Plasmas 21, (2014) 055905.

[29] Ishizawa, A., Watanabe, T.-H., Sugama, H., et.al., Nuclear Fusion 55, 043024 (2015).

[30] Ishizawa, A., Maeyama, S., Watanabe, T.-H., Sugama, H., and Nakajima, N., Journal of Plasma Physics, 81, 435810203 (2015).

[31] Watanabe, T.-H. and Sugama, H., Nuclear Fusion 46, (2006) 24.

[32] Maeyama, S., Ishizawa, A., Watanabe, T.-H., Nakajima, N., Tsuji-Iio, S. and Tsutsui, H., Computer Physics Communications, 184, (2013) 2462.

[33] Tanaka, K., and LHD experiment group, Plasma and Fusion Res. 5, S2053 (2010).

[34] Satake, S., Kanno, R., Sugama, H., Plasma and Fusion Research 3, S1062-S1062, (2008).

[35] White, A. E., Schmitz, L., McKee, G. R., et.al., Phys. of Plasmas 15056116 (2008)

[36] Holland, C., White, A. E., McKee, G. R., et.al.,Physics of Plasmas 16052301 (2009)

[37] Told, D., Jenko, F., Gorler, T., Casson, F. J., Fable, E., and ASDEX Upgrade Team, Phys. Plasmas 20, $122312(2013)$

[38] Kobayashi, S., Mizuuchi, T., Nagasaki, K., et.al., IAEA-FEC, EX/P5-13 (2008).

[39] Hazeltine, R. D. and Meiss, J. D. Plasma Confinement, Dover, Dover Pub. (2003).

[40] Li, J. and Kishimoto, Y., Phys. Plasmas 12, 054505 (2005). 


\begin{tabular}{|c|c|c|}
\hline & LHD-H & LHD-L \\
\hline$R / a$ & 6.2 & 6.2 \\
\hline$\rho=r / a$ & 0.65 & 0.68 \\
$\rho_{*}\left[10^{-3}\right]$ & 1.7 & 1.5 \\
$\nu_{i}^{*}$ & 3. & 2. \\
$\beta[\%]$ & 0.025 & 0.083 \\
$T_{e} / T_{i}$ & 0.3 & 0.2 \\
$R_{0} / L_{n}$ & 1.1 & 0.96 \\
$R_{0} / L_{T i}$ & -0.90 & 2.7 \\
$R_{0} / L_{T e}$ & 9.7 & 8.7 \\
$\hat{s}$ & 1.1 & 9.1 \\
$D_{w e l l}$ & -0.02 & 1.2 \\
Instability & ITG & ITG \\
$\gamma\left[v_{T i} / R_{0}\right]$ & 0.19 & 0.27 \\
$\chi_{i}\left[v_{T i} \rho_{T i}^{2} / R_{0}\right]$ & 13. & 11. \\
$\chi_{e}\left[v_{T i} \rho_{T i}^{2} / R_{0}\right]$ & 5.4 & 4.8 \\
$R_{0} / L_{T}-R_{0} / L_{T \text { crit }}$ & 2.4 & 2.6 \\
\hline
\end{tabular}

Table 1. The dimensionless parameters at minor radius $\rho$ : the safety factor $q$, the normalized Larmor radius $\rho_{*}$, the normalized collision frequency $\nu^{*}$, the plasma beta $\beta$, the temperature ratio $T_{e} / T_{i}$, the inverse of the gradient length $R_{0} / L_{n}$ and $R_{0} / L_{T_{s}}$, and parameters representing magnetic field structure, the magnetic shear $\hat{s}$ and the magnetic well contribution to the Mercier index $D_{\text {well }}$. In addition, simulation results including the linear growth rate $\gamma$, the inverse of the gradient length measured from the critical one $R_{0} / L_{T}-R_{0} / L_{T \text { crit }}$, the transport coefficients $\chi_{i}$ and $\chi_{e}$ are given.

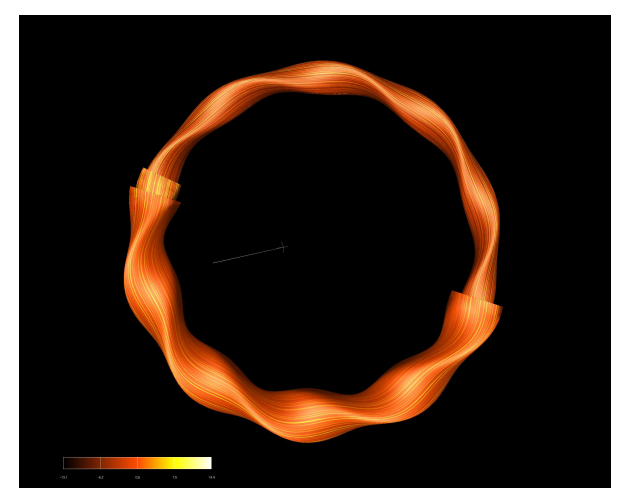

Figure 1. Top view of a magnetic surface of the LHD plasma. The striped pattern of the electrostatic potential perturbation on the surface shows the elongated mode structure of the ITG mode along the field line. 

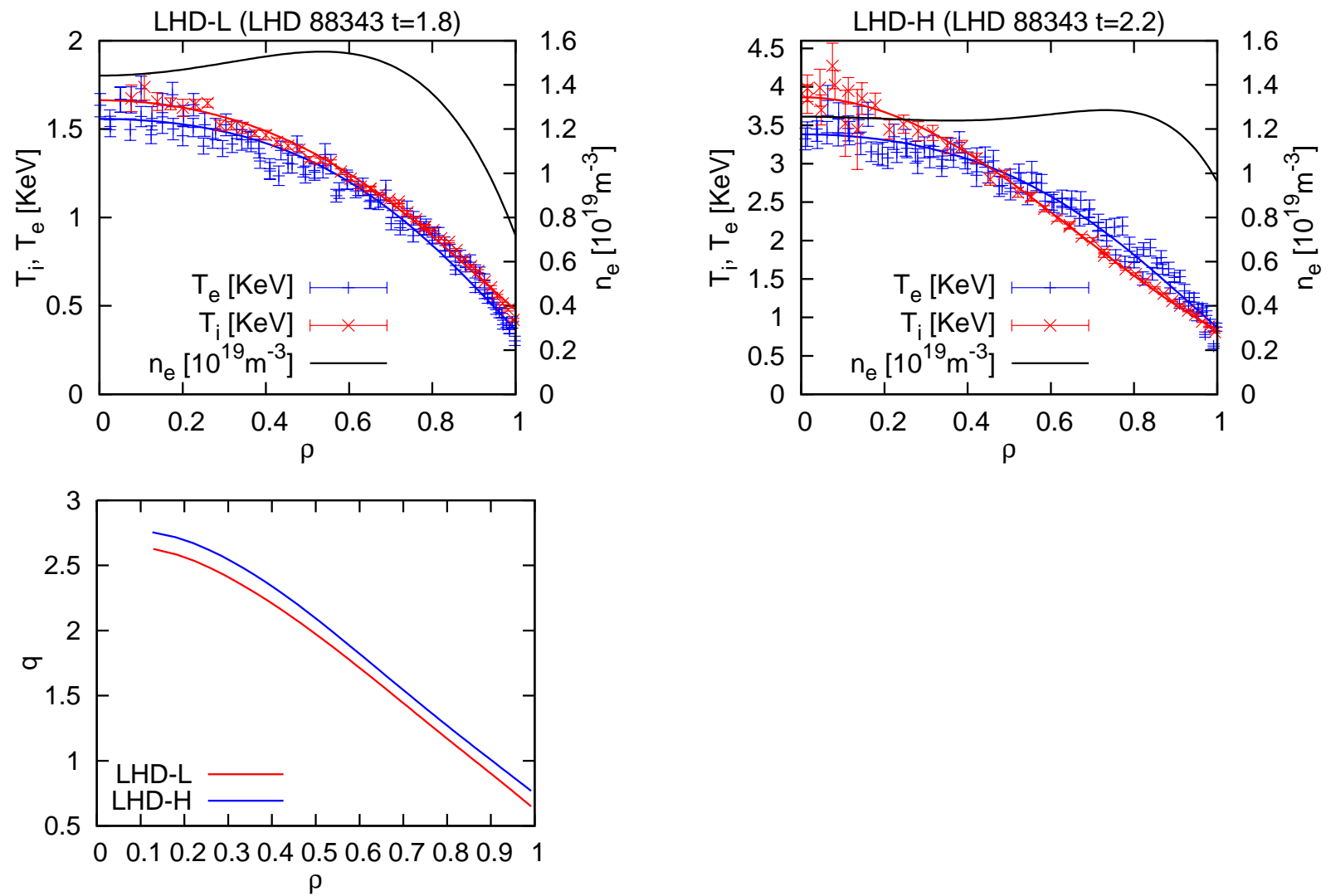

Figure 2. Profiles of ion temperature $T_{i}$, electron temperature $T_{e}$ with error bars, the electron density $n_{e}$, and safety factor $q$ of the LHD plasma with shot number 88343 before (LHD-L) and after (LHD-H) the additional NBI heating.
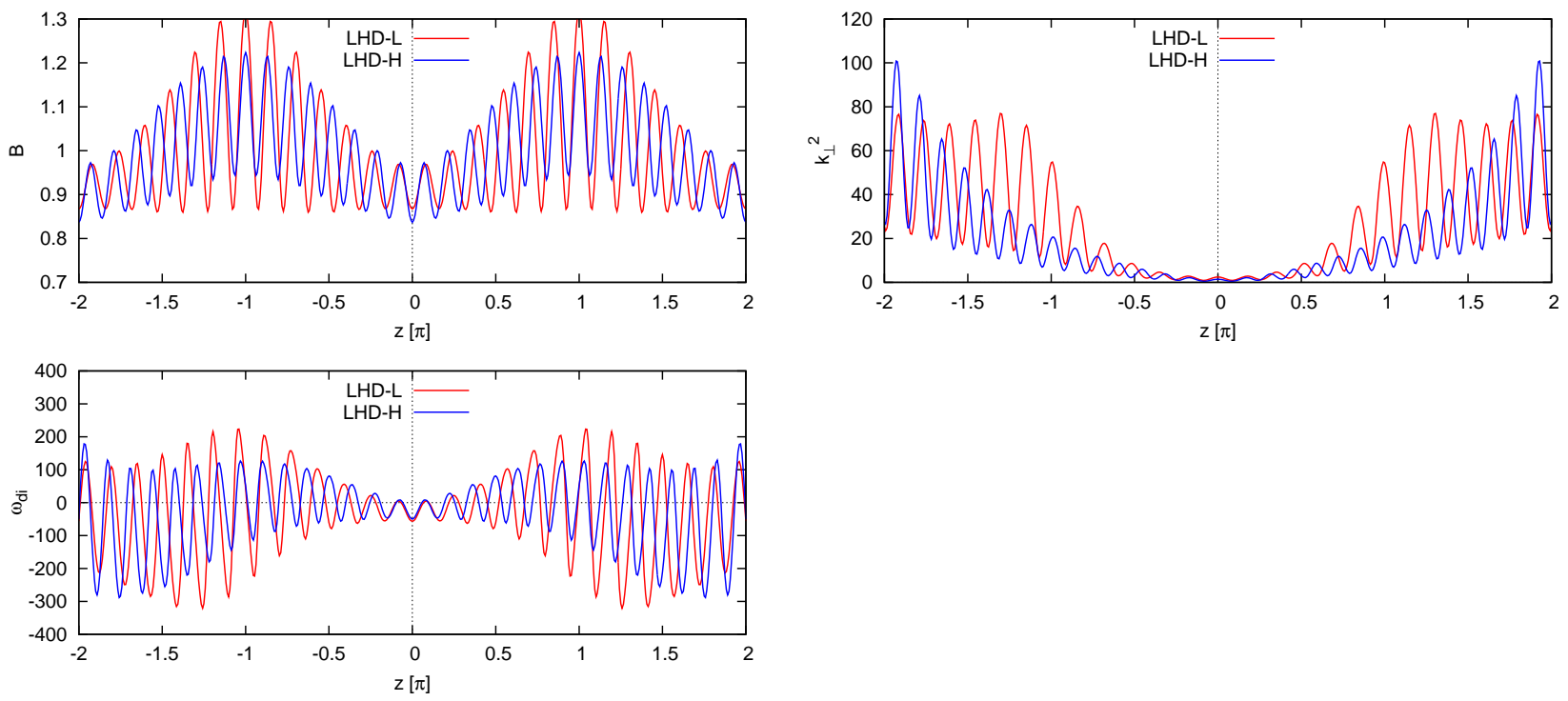

Figure 3. Profiles of magnetic field strength $B$, the normalized square of perpendicular wavenumber $k_{\perp}^{2} / k_{y}^{2}$, and the normalized magnetic drift frequency $\omega_{d i} / k_{y}$ at $\rho=0.68$ of LHD-L and at $\rho=0.65$ of LHD-H plasmas along a magnetic field line. 

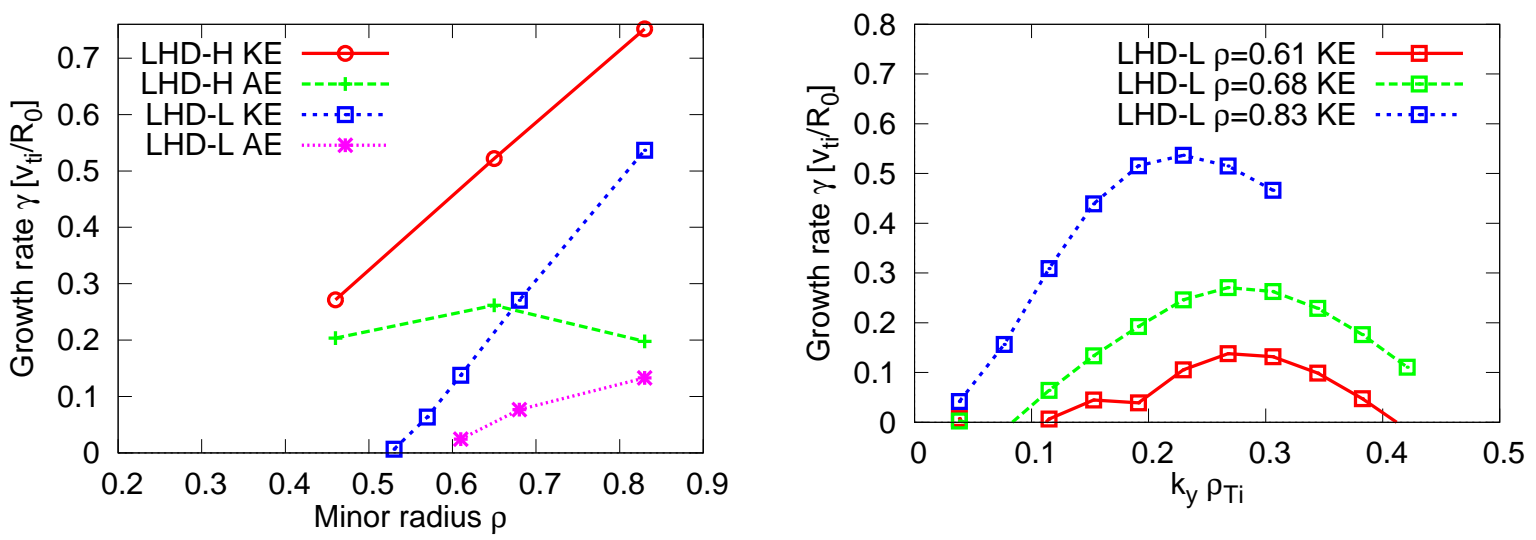

Figure 4. The linear growth rate of instabilities in the LHD-H and LHD-L plasmas. The kinetic electron (KE) effects enhance the growth rate of the ITG mode from that obtained by using the adiabatic electron (AE) approximation. The LHD-H and LHD$\mathrm{L}$ represent the plasma after the additional NBI heating at $\rho=0.65$ and the plasma before the heating at $\rho=0.68$, respectively.

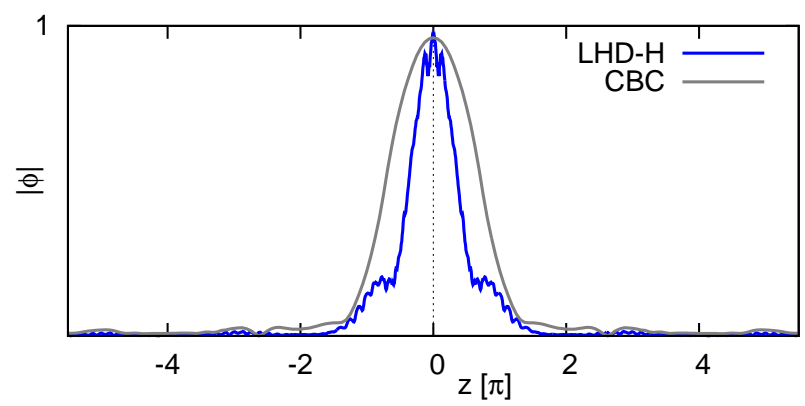

Figure 5. Electrostatic potential profile of the ITG mode along a magnetic field line in the LHD and a typical tokamak $(\mathrm{CBC})$ plasmas. The oscillation reflects the influence of particles trapped into the helical ripples.
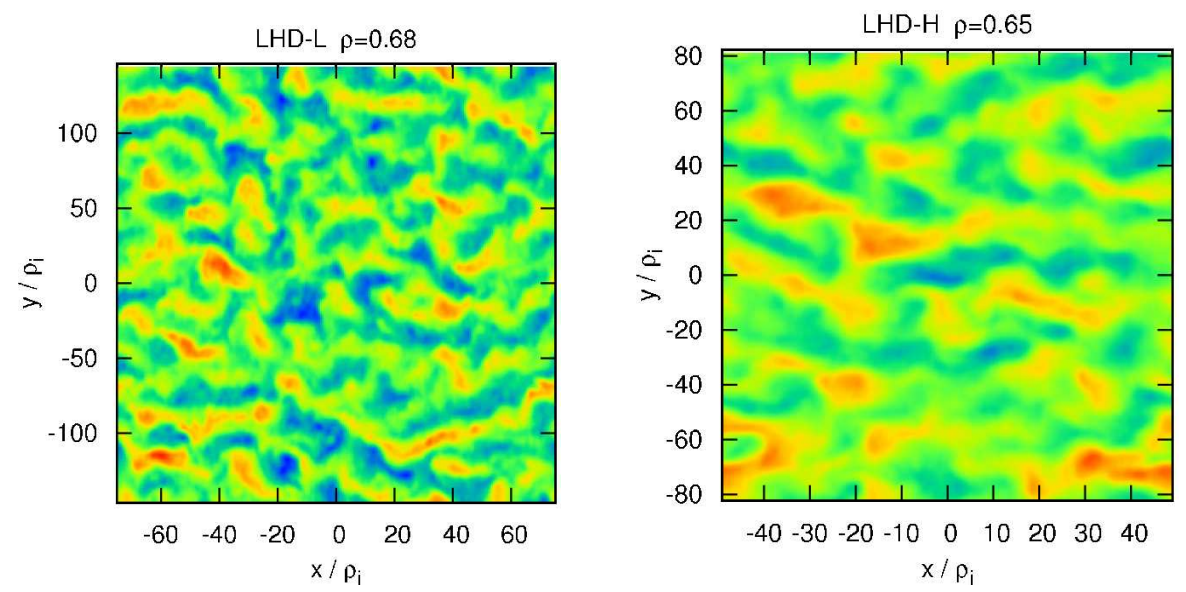

Figure 6. The profile of the electrostatic potential on the cross section at $z=0$ in the LHD-L and LHD-H plasmas. 

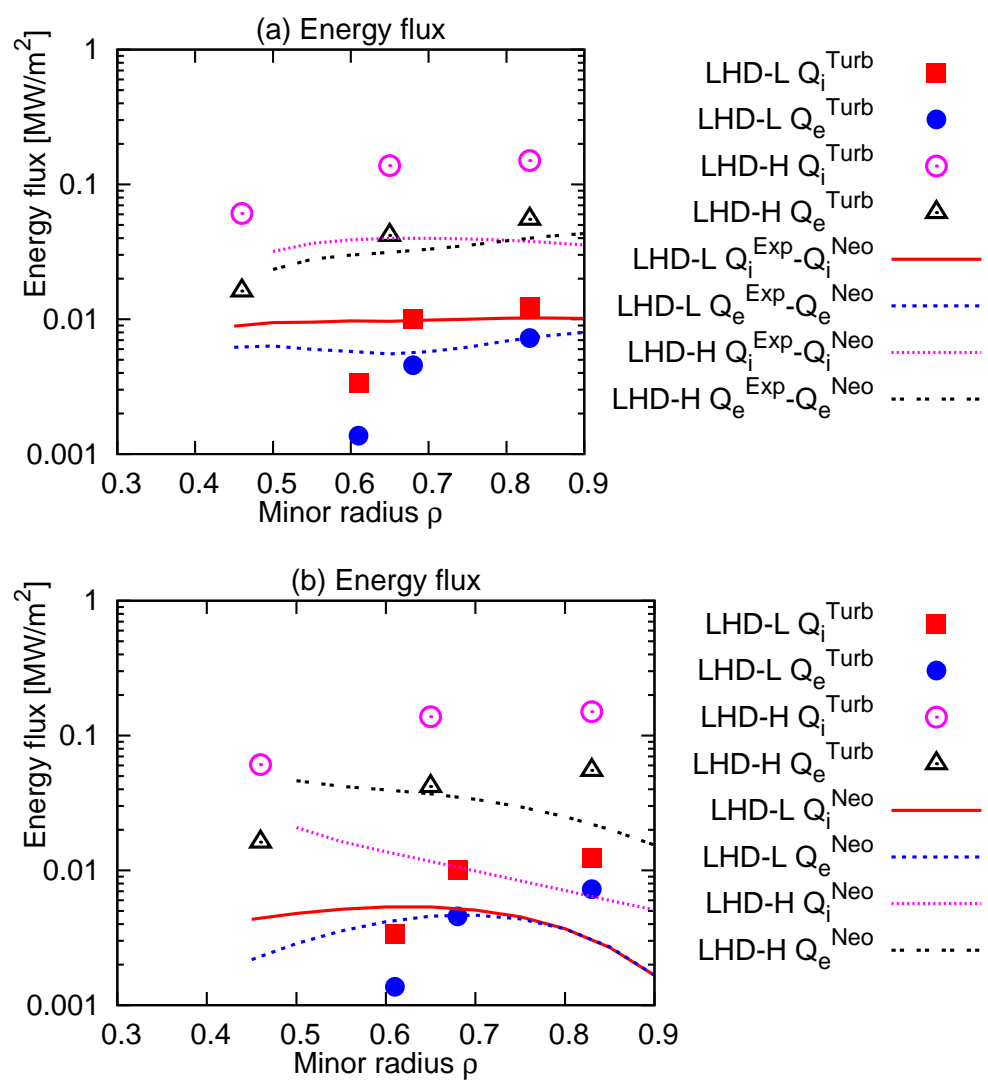

Figure 7. Turbulent ion and electron energy fluxes, in the LHD-L $(t=1.8[s]$, $\left.T_{i 0}=1.7[\mathrm{keV}]\right)$ and the LHD-H $\left(t=2.2[s], T_{i 0}=3.9[\mathrm{keV}]\right)$ plasmas. (a) symbols are from the simulations $Q_{i}^{\text {Turb }}$ and $Q_{e}^{\text {Turb }}$, and curves are from the experiments subtracted by the neoclassical flux $Q_{i}^{\text {Exp }}-Q_{i}^{\text {Neo }}$ and $Q_{e}^{\text {Exp }}-Q_{e}^{\text {Neo }}$. (b) symbols are turbulent energy fluxes $Q_{i}^{\text {Turb }}$ and $Q_{e}^{\text {Turb }}$, and curves are neoclassical energy fluxes $Q_{i}^{\text {Neo }}$ and $Q_{e}^{\text {Neo }}$.
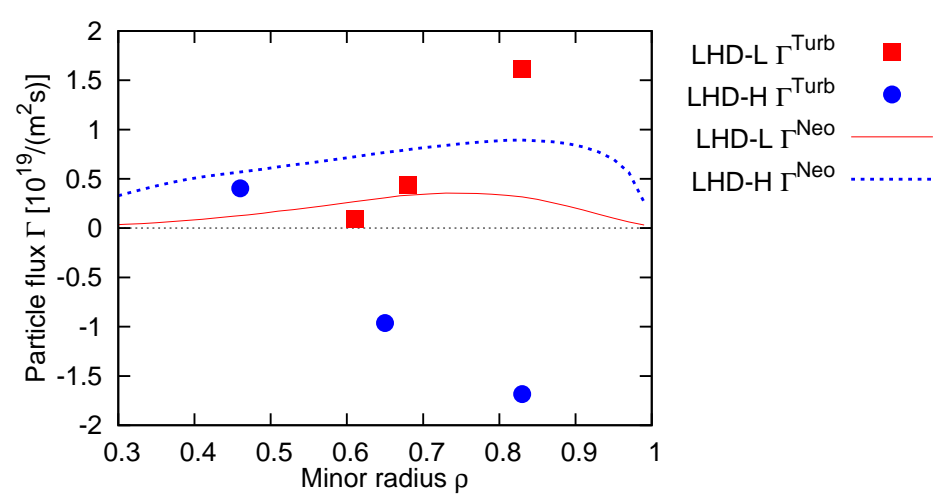

Figure 8. Turbulent particle flux and neoclassical particle flux in the LHD-L $\left(t=1.8[s], T_{i 0}=1.7[\mathrm{keV}]\right)$ and the LHD-H $\left(t=2.2[s], T_{i 0}=3.9[\mathrm{keV}]\right)$ plasmas. Symbols are turbulent flux $\Gamma^{\text {Turb }}$, and curves are neoclassical flux $\Gamma^{\text {Neo }}$. 

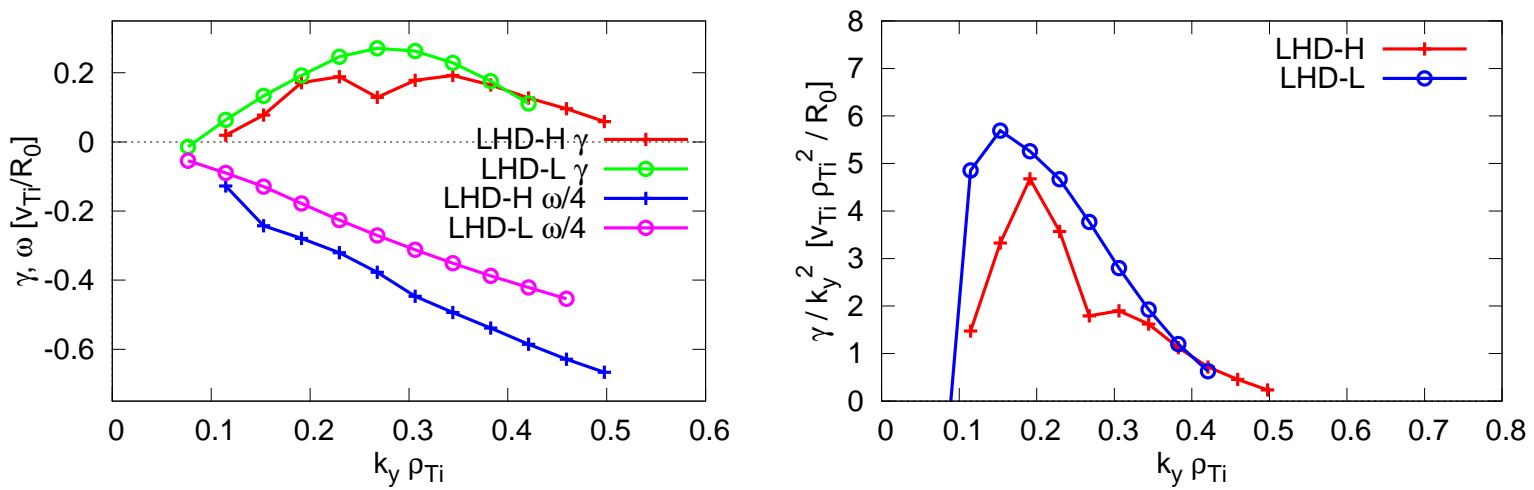

Figure 9. The linear growth rate $\gamma$, the real frequency $\omega$, and the mixing length estimated diffusion coefficient $\gamma / k_{y}^{2}$ of instabilities in the LHD plasmas as a function of the binormal wavenumber $k_{y}$.
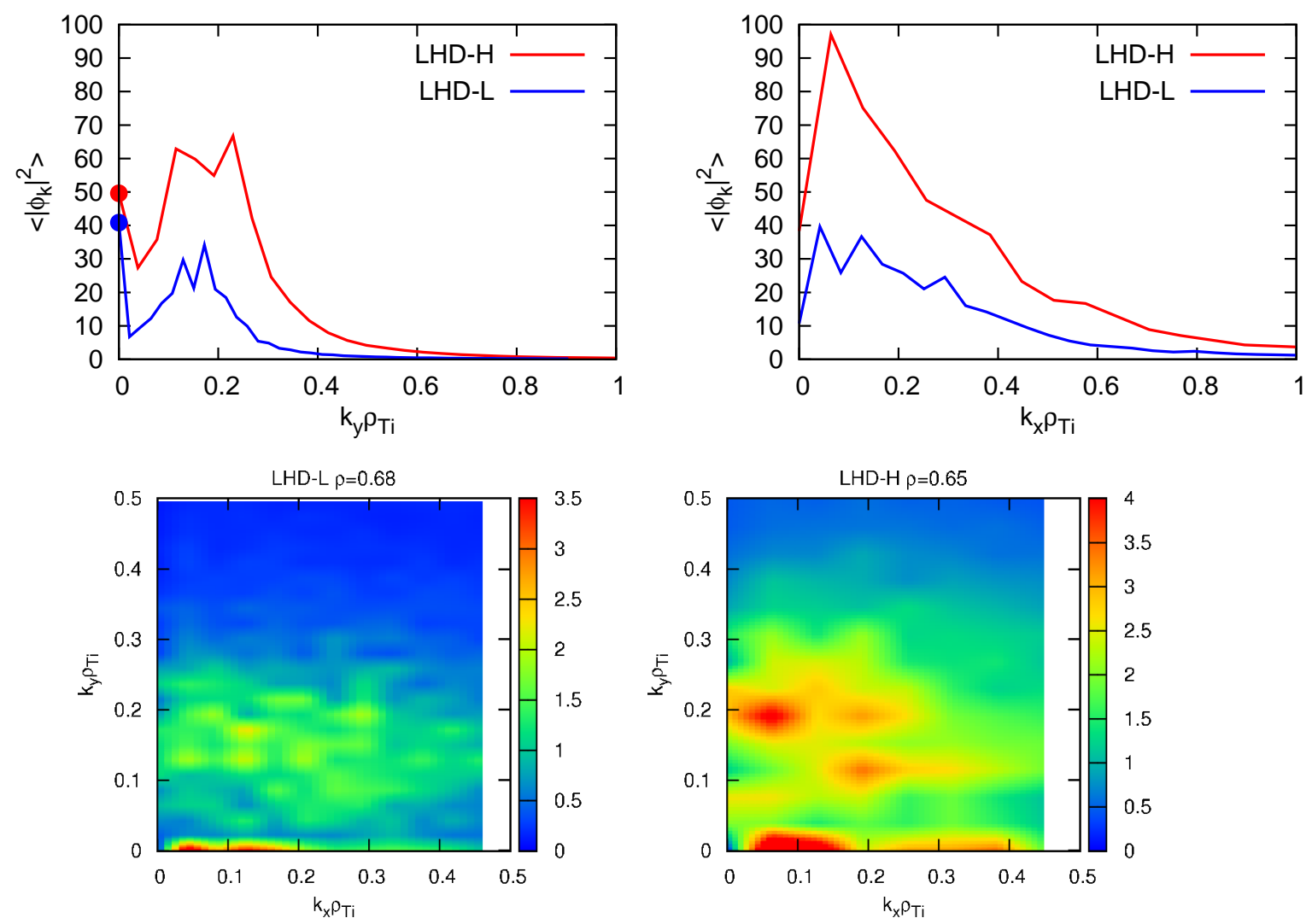

Figure 10. The spectra of the electrostatic potential $\left\langle\left|\phi_{k}\right|^{2}\right\rangle$ perturbation in the LHD$\mathrm{L}$ and LHD-H plasmas as a function of $k_{y}, k_{x}$ and in $\left(k_{x}, k_{y}\right)$ space, where $k_{x}$ and $k_{y}$ are the radial and binormal wavenumbers, respectively. The square of the zonal flow potential amplitude $\left\langle\left|\phi_{k}\left(k_{y}=0\right)\right|^{2}\right\rangle$ is represented by circles. 

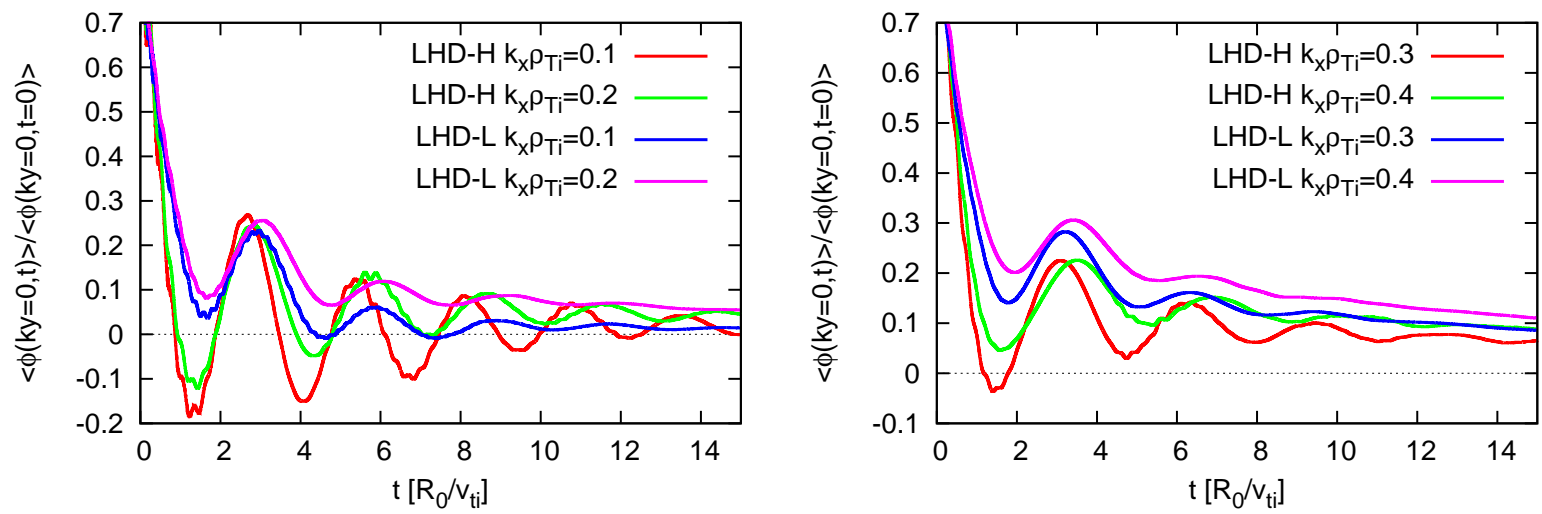

Figure 11. Time history of the normalized zonal component of the electrostatic potential $\left\langle\phi\left(k_{y}=0, t\right)\right\rangle /\left\langle\phi\left(k_{y}=0, t=0\right)\right\rangle$ for several radial wavenumbers $k_{x} \rho_{T i}=0.1$, $0.2,0.3$, and 0.4 in the LHD plasmas.

\begin{tabular}{|c|c|c|}
\hline & HJ-ST & HJ-HB \\
\hline$R / a$ & 7.3 & 7.3 \\
\hline$\rho=r / a$ & 0.5 & 0.5 \\
$q$ & 1.7 & 1.8 \\
$\rho_{*}\left[10^{-3}\right]$ & 4.5 & 4.4 \\
$\nu_{i}^{*}$ & 3.2 & 3.4 \\
$\beta[\%]$ & 0.05 & 0.05 \\
$T_{e} / T_{i}$ & 1.3 & 1.3 \\
$R_{0} / L_{n}$ & 9.3 & 9.3 \\
$R_{0} / L_{T i}$ & 13. & 13. \\
$R_{0} / L_{T e}$ & 17. & 17. \\
$\hat{s}$ & 0.023 & 0.021 \\
$D_{w e l l}$ & 0.74 & 0.64 \\
\hline Instability & ITG & ITG \\
$\gamma\left[v_{T i} / R_{0}\right]$ & 0.4 & 0.26 \\
$\chi_{i}\left[v_{T i} \rho_{T i}^{2} / R_{0}\right]$ & 5.9 & 4.2 \\
$\chi_{e}\left[v_{T i} \rho_{T i}^{2} / R_{0}\right]$ & 2.4 & 1.7 \\
$R_{0} / L_{T}-R_{0} / L_{T c r i t}$ & 5.2 & \\
\hline
\end{tabular}

Table 2. The parameters and simulation results for the standard configuration (HJST) and the high-mirror ratio configuration (HJ-HB) of the Heliotron J. 


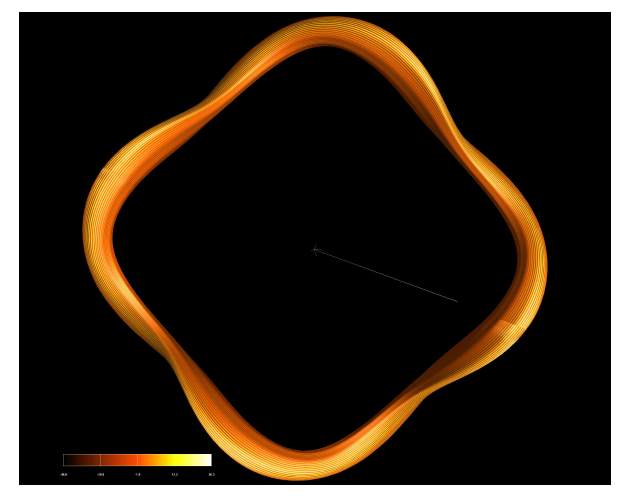

Figure 12. Top view of a magnetic surface of the Heliotron J (HJ) plasma. The striped pattern of the electrostatic potential perturbation on the surface shows the elongated mode structure of the ITG mode along the field line.
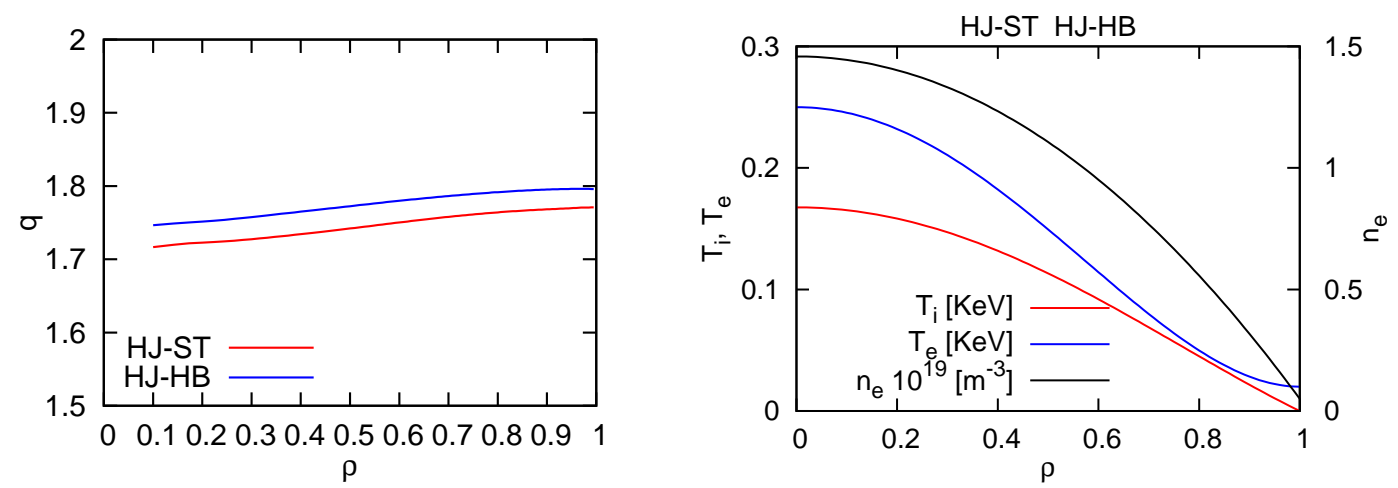

Figure 13. Profiles of safety factor $q$, the electron density $n_{e}$, and temperatures of the HJ plasmas. The HJ-ST and HJ-HB represent the standard and high toroidal mirror-ratio (high-bumpiness) configurations, respectively. 

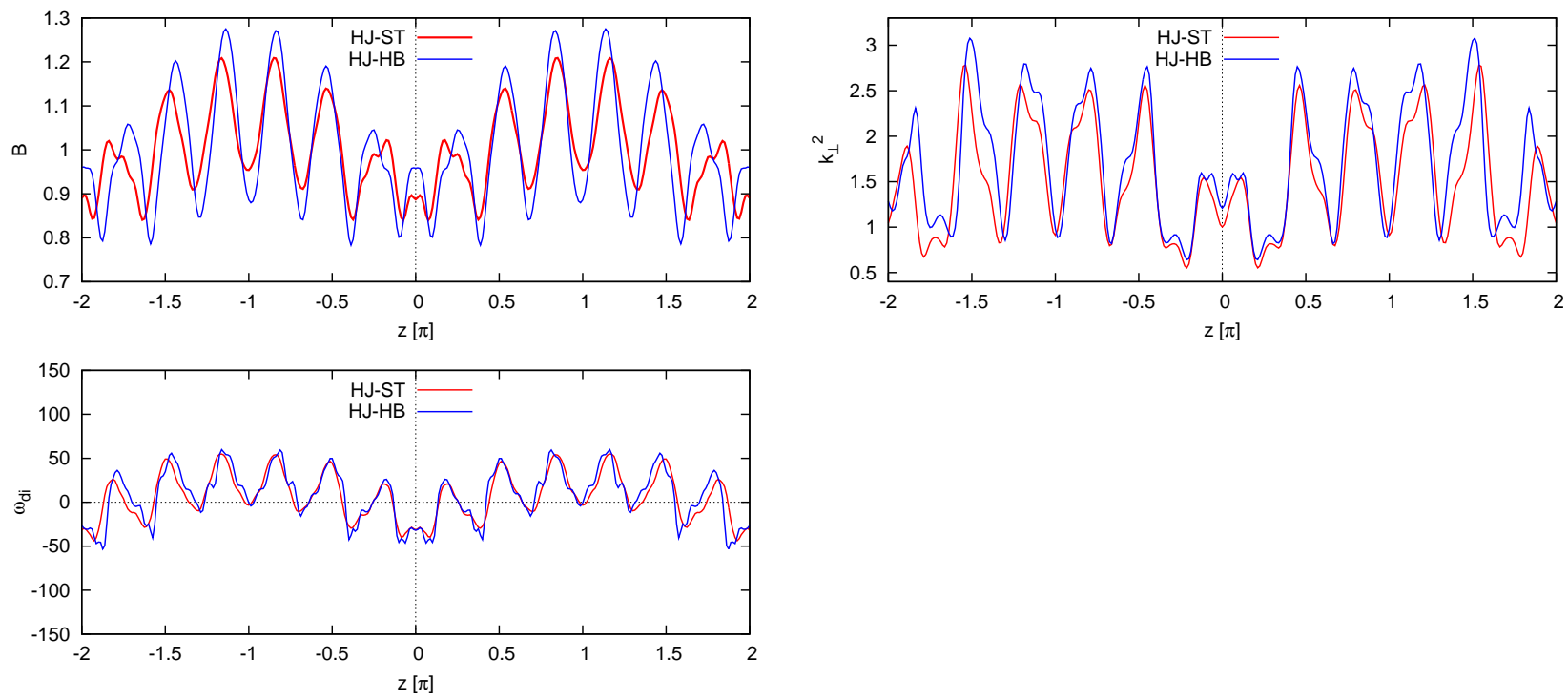

Figure 14. Profiles of magnetic field strength $B$, the normalized square of perpendicular wavenumber $k_{\perp}^{2} / k_{y}^{2}$, and the normalized magnetic drift frequency $\omega_{d i} / k_{y}$ at $\rho=0.5$ of the HJ-ST and HJ-HB plasmas along a magnetic field line.
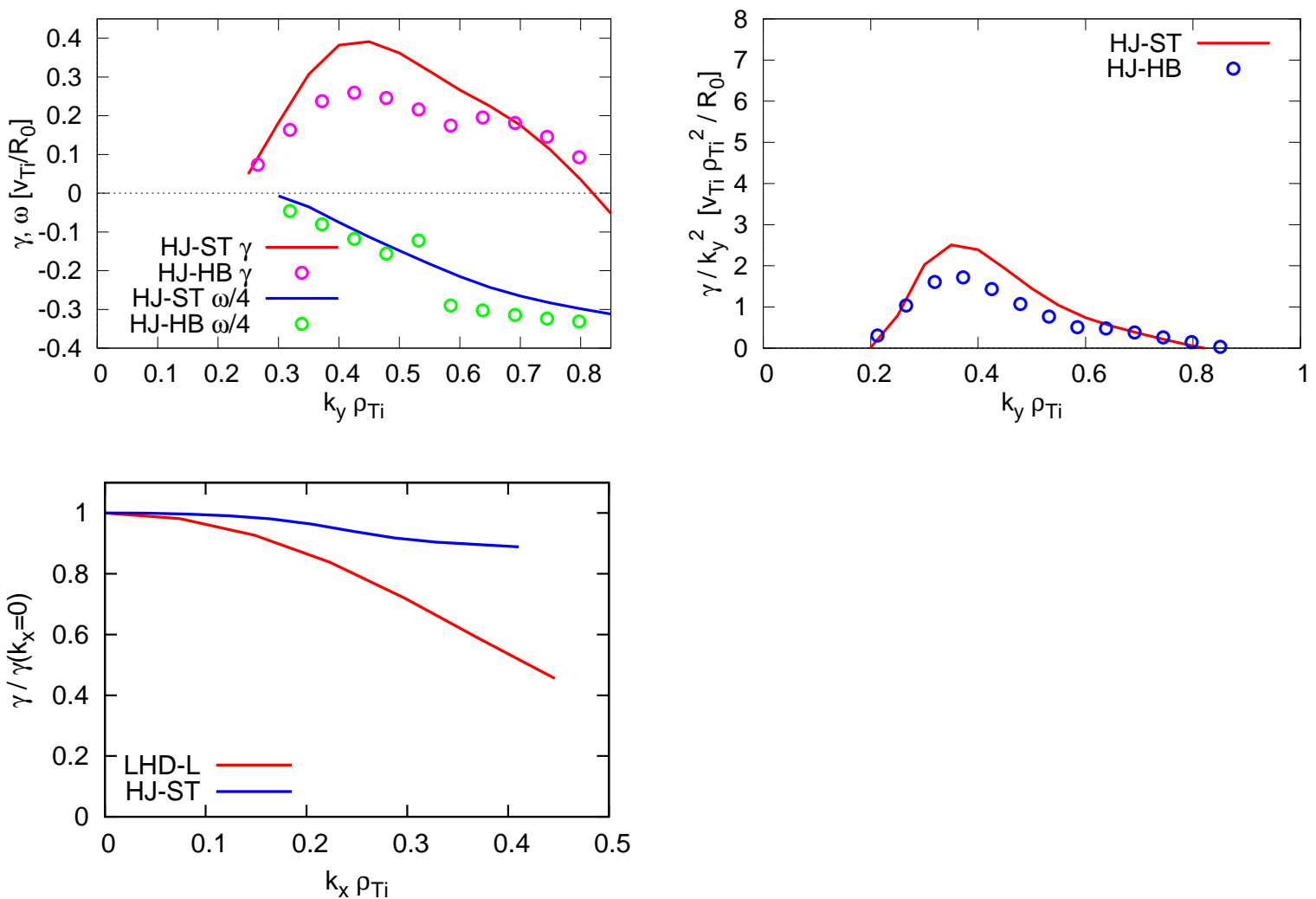

Figure 15. The linear growth rate $\gamma$, the real frequency $\omega$, and the mixing length estimated diffusion coefficient $\gamma / k_{y}^{2}$ of instabilities in the HJ plasmas as a function of the binormal wavenumber $k_{y}$, and the growth rate as a function of the radial wavenumber $k_{x}$. 


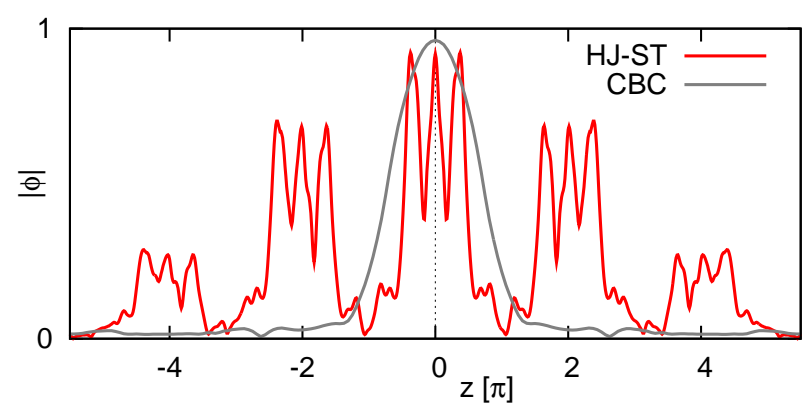

Figure 16. Electrostatic potential profile of the ITG mode along a magnetic field line in the HJ and a typical tokamak $(\mathrm{CBC})$ plasmas. The oscillation reflects the influence of particles trapped into the helical ripples.
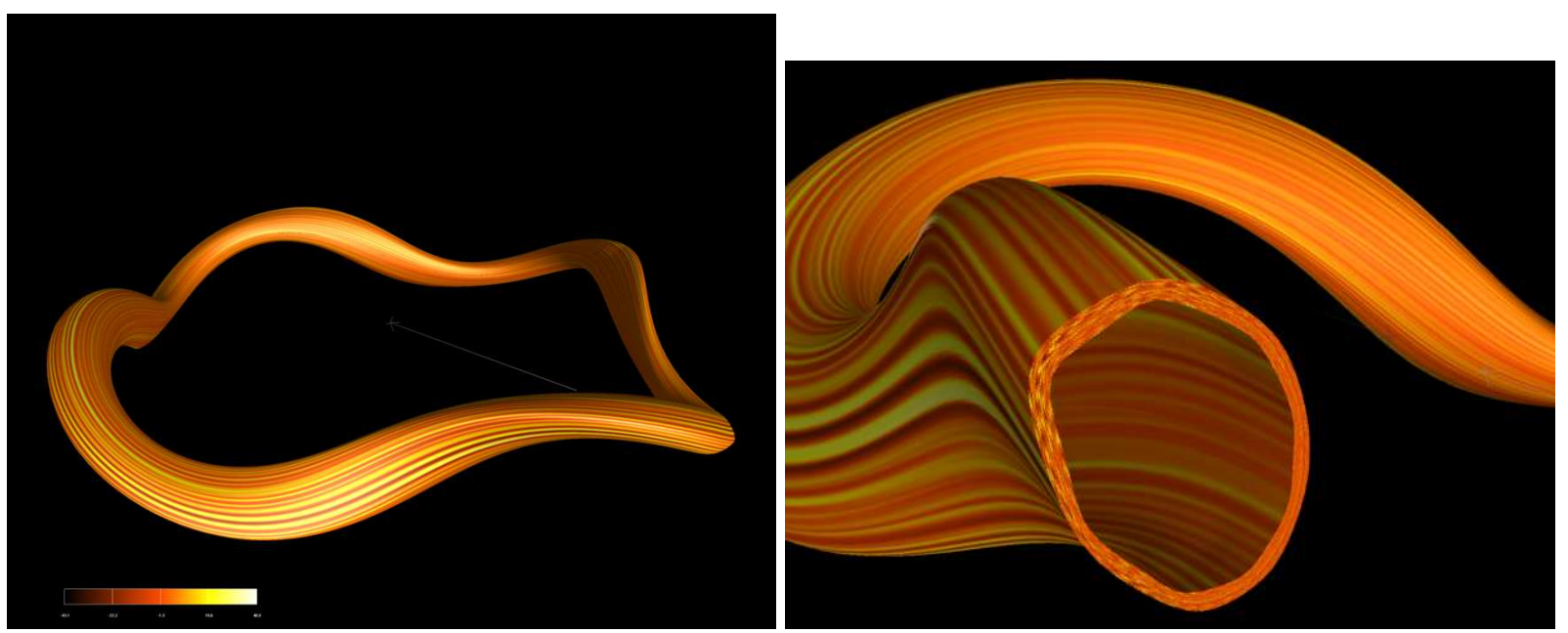

Figure 17. Three-dimensional view of the electrostatic potential perturbation of the ITG turbulence on a magnetic surface and on a cross section of the standard configuration of the HJ plasma (HJ-ST).

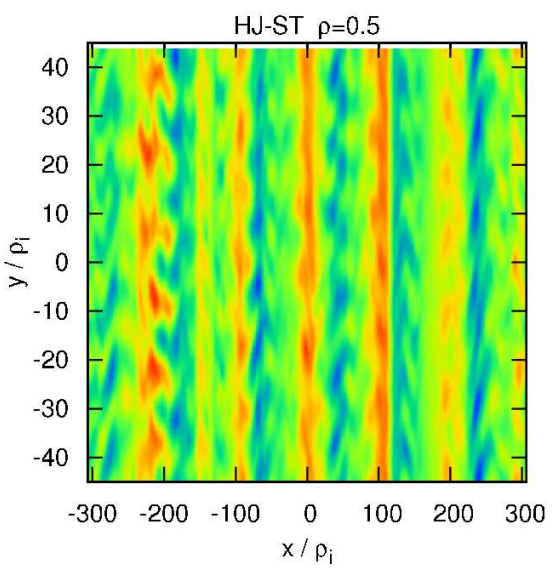

Figure 18. The profile of the electrostatic potential on the cross section at $z=0$ in the HJ-ST plasmas. 

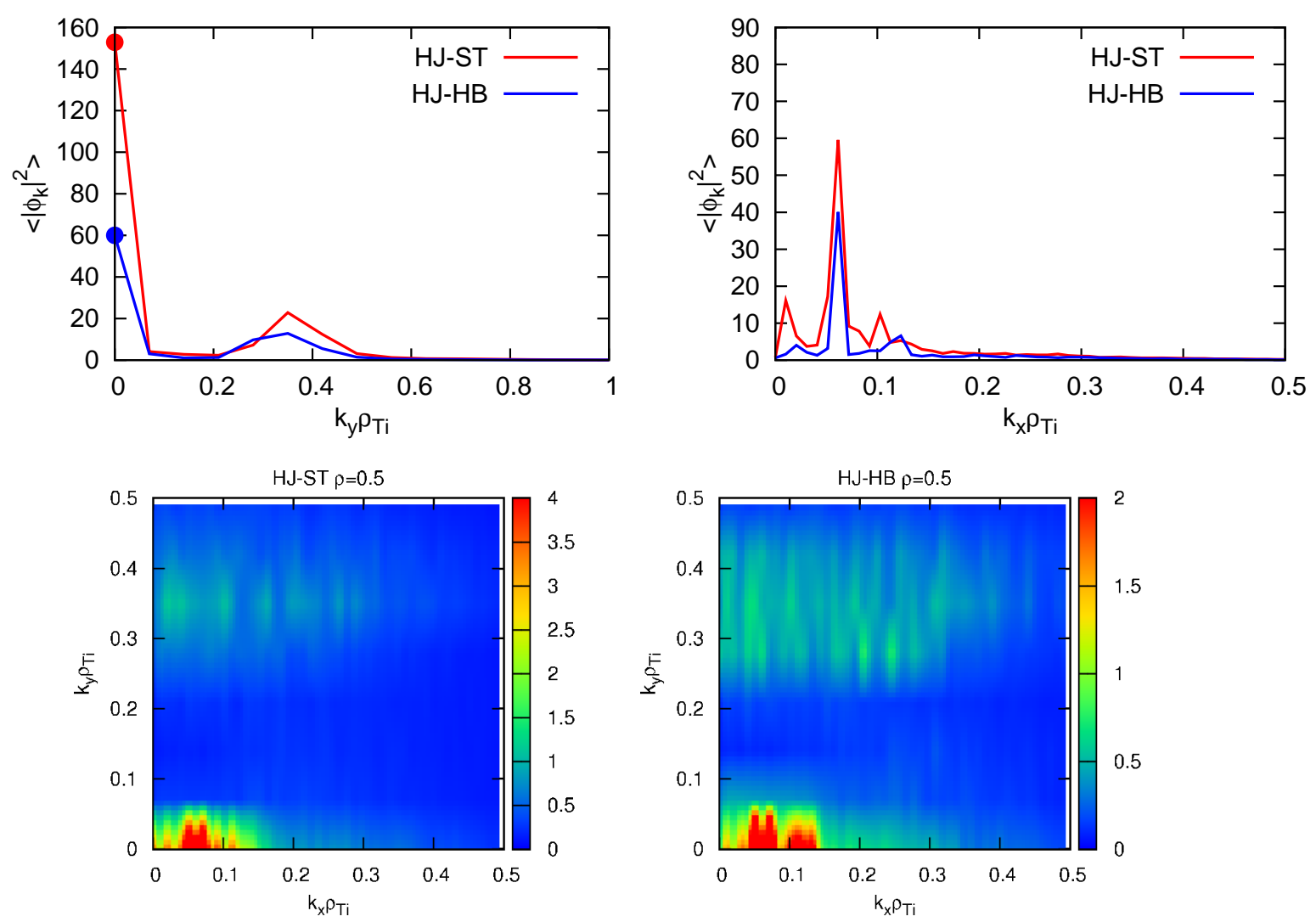

Figure 19. The spectra of the electrostatic potential $\left\langle\left|\phi_{k}\right|^{2}\right\rangle$ perturbation in the HJ$\mathrm{ST}$ and HJ-HB plasmas as a function of $k_{y}, k_{x}$ and in $\left(k_{x}, k_{y}\right)$ space, where $k_{x}$ and $k_{y}$ are the radial and binormal wavenumbers, respectively. The square of the zonal flow potential amplitude $\left\langle\left|\phi_{k}\left(k_{y}=0\right)\right|^{2}\right\rangle$ is represented by circles.
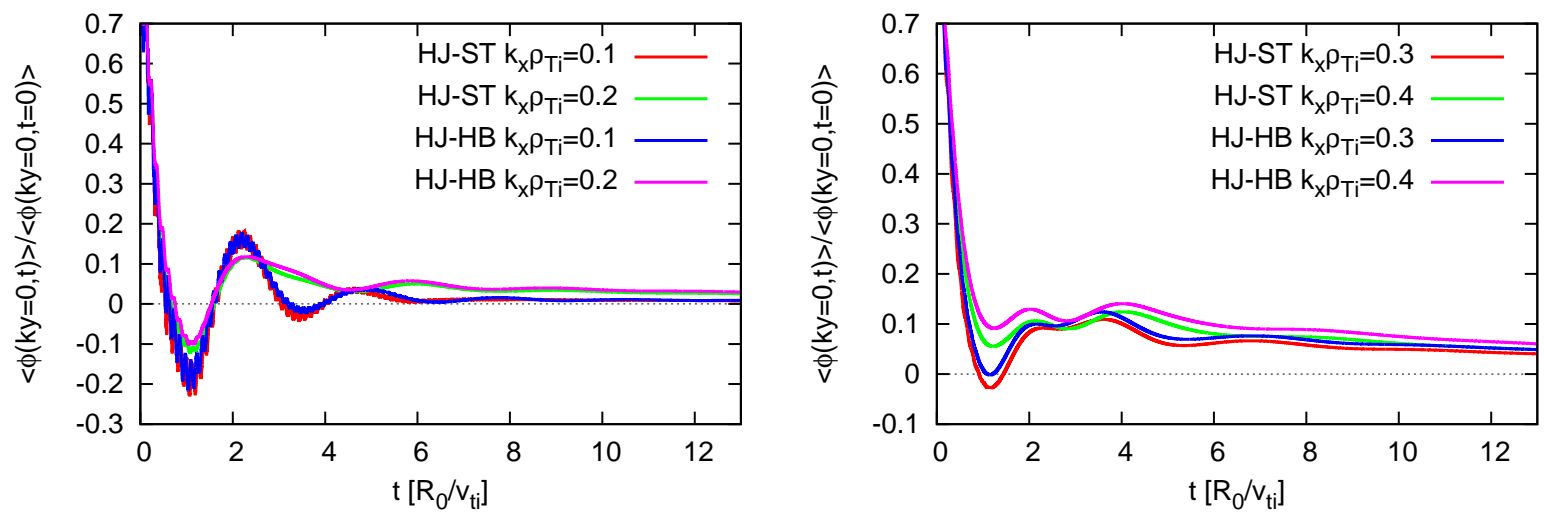

Figure 20. Time history of the normalized zonal component of the electrostatic potential $\left\langle\phi\left(k_{y}=0, t\right)\right\rangle /\left\langle\phi\left(k_{y}=0, t=0\right)\right\rangle$ for several radial wavenumbers $k_{x} \rho_{T i}=0.1$, $0.2,0.3$, and 0.4 in the HJ plasmas. 